\title{
La morale dans l'office du juge constitutionnel en Afrique francophone
}

\author{
H. N'Sinto A. T. Lawson*
}

\section{Résumé}

Le rôle de pacification du débat politique que devrait jouer le juge constitutionnel en Afrique francophone est encore incertain. Critiqué pour sa prétendue proximité du pouvoir politique, le juge constitutionnel africain dans son office est parfois contraint de recourir à la règle morale. Cette contribution transcende le débat sur le pouvoir normatif du juge pour évaluer la portée de la règle morale sur les décisions de justice constitutionnelle. D'une part, il s'agit d'analyser la fabrique des décisions de justice à l'aune de la morale. Cette analyse porte respectivement sur les occurrences de la morale dans la décision du juge constitutionnel et les circonstances du recours à la morale dans les jurisprudences ordinaires. Elle révèle la prudence avec laquelle le juge, constitutionnel ou ordinaire, convoque la morale dans son office. D'autre part, il est constaté dans la convocation de la morale dans l'office $\mathrm{du}$ juge, une transformation de la fonction prétorienne. Le juge constitutionnel africain se mue en un pouvoir législatif concurrent entraînant parfois une insécurité juridique. Enfin, cette réflexion s'ouvre sur la question de la moralisation de la vie politique en Afrique et relève que l'office du juge est confronté au mythe du gouvernement des juges.

\section{Summary}

The pacification role of the political debate that the constitutional judge should play in French-speaking Africa is still uncertain. Criticized for his alleged proximity to political power, the African constitutional judge in his office is sometimes forced to resort to moral rule. This contribution transcends the debate about the normative power of the judge to assess the scope of the moral rule on constitutional court decisions. On the one hand, it is a question of analyzing the fabric of judicial decisions in the light of morality. This analysis deals respectively with the occurrences of morality in the decision of the constitutional court and the circumstances of the use of morality in ordinary case law. It reveals the prudence with which the judge, constitutional or ordinary, summons morality in his office. On the other hand, it is noted in the convocation of morality in the office of the judge, a transformation of the praetorian function. The African constitutional judge turns into a competing legislative power, sometimes leading to legal uncertainty. Finally, this reflection opens

* Docteur en droit, enseignant-chercheur, Faculté de Droit, Université de Lomé, Togo (nslawson79@gmail.com). 
on the question of the moralization of the political life in Africa and notes that the office of the judge is confronted with the myth of the government of the judges.

\section{Introduction}

"Souvent, les analyses de la tradition juridique occidentale admettent comme un acquis l'hypothèse de la séparation de la morale et du droit. Et c'est, bien sûr, sur cette base que les interférences que l'on voit survenir entre les deux domaines paraissent alors à chaque fois problématiques, en particulier parce qu'elles semblent prêter aux juges des pouvoirs qui ne leur reviennent pas $» .^{1}$ Les relations entre l'acte de juger et la morale engendrent toute une série de difficultés récurrentes. Des difficultés qui expliquent le choix de la réflexion sur « La morale dans l'office du juge constitutionnel en Afrique francophone ». ${ }^{2}$ Sans doute, la thématique n'est pas nouvelle. Le sujet avait déjà été discuté et rediscuté dans une approche plus générale. ${ }^{3}$ Champeil-Desplats expliquait sur le «droit jurisprudentiel dérobé », que le juge est sorti de son rôle et est allé au-delà de ce qu'on suppose être sa fonction. ${ }^{4}$ Le philosophe du droit Dworkin avait déjà admis que le juge doit être un juge idéal, « un Hercule », capable de donner pour tous les cas qui se présentent à lui une bonne réponse, ${ }^{5}$ par application de la règle immédiatement disponible dans les cas jugés faciles, ou par recours à des principes implicites déduits des valeurs morales, sociales ou philosophiques. $^{6}$

L'étymologie du mot « morale » vient du latin Moralis, traduction par Cicéron du grec ta èthica qui désigne ce qui a trait aux mœurs et de façon générale, aux règles de conduite. La morale se présente comme un ensemble d'idées à " caractère normatif » permettant d'éclairer et de justifier les actions humaines en les associant à la recherche du bien et du

1 GENARD (J.-L.), « Droit, éthique et responsabilité », Revue internationale d'éthique sociétale et gouvernementale, vol., 3, $\mathrm{n}^{\circ} 2,2001$, https://ethiquepublique.revues.org/2507 consulté le 25 juin 2017.

2 DOSSO (K.), « Les pratiques constitutionnelles dans les pays d'Afrique noire francophone : cohérences et incohérences ", Revue française de droit constitutionnel, 2012/2 n 90, page 57. Voir aussi, OIF, Rapport sur l'état des pratiques de la démocratie, des droits et des libertés - 2016, Dynamiques constitutionnelles dans l'espace francophone, https://www.francophonie.org/IMG/pdf/rap port-dapg-web.pdf consulté le 27 juillet 2019.

3 Lors d'un Colloque qui s'est déroulé les 25 et 26 janvier2006 à l'Institut de France, sur « La jurisprudence. La création du droit par le juge », AVRIL (P.) expose : « La jurisprudence institutionnelle du Conseil constitutionnel est-elle créatrice de droit? ». MATHIEU (B.), quant à lui communique sur « Les rôles du juge et du législateur dans la détermination de l'intérêt général ».

4 «Le discours sur le droit jurisprudentiel dérobé » in Le droit dérobé, Actes du Colloque de Montpellier 2003, Grands Colloques, Montchrestien, 2007, p.112.

5 Selon l'analyse de ALLARD Julie dans sa contribution intitulée « Dworkin : une philosophie critique du jugement », publiée à la Revue internationale de philosophie 2005/3 (n 233), p. 327; Dworkin considère que la tâche qui incombe au juge semble surhumaine, au point que seul Hercule est en mesure de l'accomplir.

6 CHAMPEILS-DESPLATS (V.), op. cit. 
juste. ${ }^{7}$ Elle est distincte du droit, mais n'est pas ignorée par le droit car ce dernier se fonde généralement sur des considérations de justice et de bien social. Cependant, le recours à la morale dans l'argumentation juridique est souvent discuté, car l'on y voit un carcan contribuant à l'assujettissement social des individus. ${ }^{8}$ La morale et le droit auraient des origines différentes. ${ }^{9}$ Dans sa très intéressante étude sur la comparaison des règles morales et juridiques, Dabin entend les règles juridiques exclusivement au sens de règles du droit positif. On sait d'ailleurs que, pour lui, seul ce droit est véritablement juridique. ${ }^{10}$ Pour Ripert, il n'y aurait pas de différence de nature ou de but entre la règle de droit et la règle morale. ${ }^{11}$ Ainsi, l'on peut rapprocher le concept de la morale d'autres concepts, notamment l'éthique, ${ }^{12}$ l'équité, ${ }^{13}$ la moralité, ${ }^{14}$ et bien d'autres concepts comme la cause, la bonne foi, la dignité, l'abus de droit... Des concepts auxquels le juge se réfère régulièrement dans son interprétation, mais dont le législateur donne rarement de définition. Des notions, il faut le souligner, qui ne datent pas d'aujourd'hui, ni d'hier, et pour reprendre les propos de Antigone à Créon, « sont éternelles et personne ne sait à quel passé elles remontent $» .{ }^{15}$ En prenant comme " témoin », le concept d'éthique, l'on note que celui-ci a la même étymologie que la morale, puisque les deux renvoient aux mœurs. ${ }^{16}$ Les termes " morale » et « éthique » sont souvent confondus. ${ }^{17}$ Il est important d'indiquer que les doctrines positi-

7 Elle est censée guider les conduites des individus dans leur vie personnelle comme dans leur vie sociale. La morale est habituellement valorisée dans les idéologies conservatrices qui y voient un ferment de l'unité de la société.

8 NAY (O). et autres, Lexique de science politique, vie et institutions politiques, Dalloz, 2008, p. 330.

9 Le domaine de la morale est étendu et englobe à la fois les rapports à autrui, mais aussi les rapports à soi-même, voire à une Divinité. Le droit est censé être moralement neutre et édicte les règles nécessaires à la vie en société. La morale régit également la pensée et les envies alors que le droit s'en désintéresse. La morale est destinée à améliorer l'individu, il s'agit fréquemment d'un idéal à atteindre alors que le droit est destiné à être respecté par tous.

10 Par règle morale, il entend celle qui prétend diriger les êtres humains dans le sens d'un certain idéal de vie. DABIN (J.), « La technique de l'élaboration du droit positif ». In: Revue néo-scolastique de philosophie. $40^{\mathrm{e}}$ année, Deuxième série, $\mathrm{n}^{\circ} 54,1937$, p. 288

11 RIPERT (G.), La règle morale dans les obligations civiles, LGDJ, 1949, ${ }^{\circ} 13 * 18$. Il soutenait que le droit, même dans ses parties les plus techniques, est toujours dominé par la loi morale.

12 Pour Aristote, l'éthique est la science du bonheur humain, et il énumère les biens qui, à ses yeux, constituent les parties du bonheur humain.

13 L'équité est la réalisation suprême de la justice, allant au-delà de ce que prescrit la loi...

14 L'article 42 du statut de Rome, prévoit l'indépendance du procureur et exige les qualités morales et une grande expérience en matière d'instruction et de poursuite ou de procès au pénal.

15 MONSENGWO (L.), La notion de nomos dans le pentateuque grec, Editrice Pontificio Istituto Biblico, 2005, p. 35

16 Mores en latin, ethos en grec

17 NAY (O). et autres, Lexique de science politique, vie et institutions politiques, Dalloz, 2008, p. 330. Selon le Lexique de science politique rédigé sous la direction de Nay, la morale est associée à 
vistes, avec Kelsen, conçoivent le droit dans un état de pureté parce qu'expurgé de toute morale. ${ }^{18}$

Aujourd'hui, cette controverse n'intéresse qu'assez peu le point de vue épistémologique. Dans la même perspective, Robespierre dit, «qu'il faut des lois précises et point de jurisprudence ». Une opinion sans doute rigide mais qui se veut le chantre du principe de légalité des peines. Une opinion contre laquelle, Portalis se démarquera rapidement et affirmera que "la loi ne peut tout prévoir et tout dire ». ${ }^{19}$ Pour Portalis, "c'est au magistrat et au jurisconsulte, pénétrés de l'esprit général des lois, d'en diriger l'application $\gg .{ }^{20}$ Traiter de la morale dans l'office du juge constitutionnel, nécessite de préciser le concept de l'office du juge.

L'expression « office du juge » fait partie de ces termes juridiques difficile à définir, car elle accumule les sens et tend à devenir inaudibles. Si elle survit, c'est grâce à son imprécision. ${ }^{21}$ Selon, le Vocabulaire juridique de Cornu, le mot « office » signifie : « Ensemble des devoirs et des pouvoirs attachés à une fonction publique $»{ }^{22}$ Aussi, l'on peut en déduire que l'office du juge ne serait donc qu'un ensemble de devoirs et de pouvoirs attachés à la fonction de juger. Frison-Roche considère qu'il s'agit tout à la fois de trancher le litige, d'apaiser le conflit, d'appliquer la loi et de réaliser la vertu de justice. ${ }^{23} \mathrm{Il}$ ne peut s'agir que de devoirs et de pouvoirs déterminés par une source normative dite positive, c'est-à-dire ayant par convention une nature contraignante. ${ }^{24}$ Mais aujourd'hui, nul ne peut plus sérieusement soutenir que le juge ne soit que l'oracle de la loi. ${ }^{25}$ Le juge va au-delà de la loi, va l'interpréter et recourt aussi à la règle morale. C'est reconnaître à l'instar de Remy que le juge n'est plus «sous la loi », ni même «à côté de la loi », il est « au-dessus de la loi », ${ }^{26}$ une formule sans doute un peu audacieuse.

des prescriptions collectives ayant un effet contraignant sur les personnes, alors que l'éthique est présentée comme des règles et des principes choisis par les individus pour guider leurs conduites. L'éthique demeure un concept mou et doux.

18 KELSEN H., Théorie pure du droit (traduction française par Ch. Eisenmann). In: Revue internationale de droit comparé. Vol. 15 N4, Octobre-décembre 1963. p. 794-795.

19 FOYER (J.), Allocution d'ouverture du Colloque sur « La jurisprudence. La création du droit par le juge » Académie des sciences morales et politiques, Université Paris XIII, 2006.

20 Idem.

21 OURLIAC (P.), "L'office du juge dans le droit canonique ", Mélanges offerts à Pierre HEBRAUD, Toulouse, Presses de l'université des sciences sociales, 1981, p. 627.

22 CORNU (G.), Vocabulaire juridique, Paris, PUF, 2008 (1987), p. 633.

23 FRISON-ROCHE (M.A.), « Les offices du juge », in Mélanges Jean Foyer, PUF, 1997, p. 463.

$24 \mathrm{La}$ Constitution, la loi, le règlement, mais aussi d'autres textes internationaux, tels que la Charte africaine des droits de l'homme, la Convention européenne de sauvegarde des droits de l'homme et des libertés fondamentales. BERNABE (B.), «L'office du juge et la liturgie du juste », Cahiers philosophiques 2016/4 ( $\mathrm{n}^{\circ} 147$ ), p. 49.

25 Voir, «Les juges », Revue Pouvoirs, ${ }^{\circ} 74$.

26 REMY (P.), « La part faite au juge », Pouvoirs 2003/4 (n 107), p. 22-36. 
Il importe de préciser que dans le cadre de cette contribution, il s'agira de l'office du juge constitutionnel en Afrique francophone ${ }^{27}$. Qu'est donc un juge constitutionnel? Les réponses sont nombreuses et varient d'un auteur à un auteur et d'une école à une autre. Aux avis des spécialistes du droit constitutionnel, il faut mentionner ceux des spécialistes du droit administratif et du droit privé. En somme, la qualité du juge constitutionnel est surtout reconnue au juge de la juridiction constitutionnelle ${ }^{28}$ mais aussi au juge protecteur des droits de la minorité ${ }^{29}$ et enfin au juge défenseur de la souveraineté du peuple. ${ }^{30}$ Le juge constitutionnel est un des acteurs principaux de la justice constitutionnelle, celle-ci désignant, selon Favoreu, "l'ensemble des institutions et techniques grâce auxquelles est assurée sans restriction, la suprématie de la constitution $» .^{31}$ Mais selon, Rubio Llorente, ${ }^{32}$ il existe deux conceptions de la justice constitutionnelle. Une conception subjective selon laquelle la juridiction constitutionnelle est celle qu'exercent les cours constitutionnelles quelle que soit la nature des affaires examinées par elles. Une conception matérielle qui assimile juridiction constitutionnelle et contrôle juridictionnel de la constitutionnalité. Cette précision importe dans les études sur le constitutionnalisme en Afrique. ${ }^{33}$ Car l'on relève une forte apparition des juridictions constitutionnelles spécialisées ${ }^{34}$ depuis le début des années $1990 .{ }^{35}$

27 Voir HOLO (T.), « Emergence de la justice constitutionnelle », in Revue Pouvoirs, n 129, 2009, p. 101-114, et CONAC Gérard, "Le juge de l'Etat en Afrique francophone », MANGIN Gilbert, "Quelques points de repères dans l'histoire de la justice en Afrique francophone », in sous dir. Jean du Bois de GAUDUSSON et CONAC Gérard.

28 Conseil constitutionnel en France, Cour constitutionnelle au Togo.

29 Voir BRUNET P., « Le juge constitutionnel est-il un juge comme les autres? Réflexions méthodologiques sur la justice constitutionnelle » in Actes du colloque la notion de "justice constitutionnelle », Dalloz, 2005, p. 125.

30 ROUSSEAU (D.), « La justice constitutionnelle : quelle nécessité démocratique? », in MOLFESSIS et al. (sous la dir. de), La légitimité du Conseil constitutionnel, Paris Economica, 1999, p. 370.

31 FAVOREU (L.) et alii, Droit constitutionnel, Dalloz, Paris, 1999, p. 185. Voir aussi, de VILLIERS (M.) et le DIVELLEC (A.), Dictionnaire du droit constitutionnel, Sirey, 2015, p. 206.

32 RUBIO LLORENTE (F.), Tendances actuelles de la juridiction constitutionnelle en Europe, AIJC, 1996, p. 13.

33 CABANIS (A.), MARTIN (M.-L.), Les Constitutions d'Afrique francophone. Évolutions des Constitutions récentes, Paris, L'Harmattan, 1999. Voir GLELE (A. M.), « La Constitution ou loi fondamentale », in Encyclopédie juridique de l'Afrique, Abidjan-Dakar-Lomé, Les nouvelles Éditions africaines, 1982, p. 33-34. Voir aussi OWONA (J.), « L'essor du constitutionnalisme rédhibitoire en Afrique noire. Étude de quelques "constitutions Janus" ", in Mélanges P.-F. Gonidec, État moderne : horizon 2000 : aspects interne et externe, Paris, LGDJ, 1985, p. 235.

34 Voir, OULD BOUBOUTT (A. S.), « Les juridictions constitutionnelles en Afrique. Evolutions et enjeux. », in Annuaire international de justice constitutionnelle, 13-1997, 1998, 1445.

35 Il existe des juridictions ordinaires qui possèdent outre leurs compétences classiques, celle du contrôle de constitutionnalité. Voir HOUNAKE (K.)., Les juridictions constitutionnelles dans les Démocraties émergentes de l'Afrique noire francophone: les cas du Bénin, du Gabon, du Niger, du Sénégal et du Togo, Thèse de Doctorat en Droit public, Université de Lomé-Université de Poitiers, 06 avril 2012, 625 p. 
Saisir la justice constitutionnelle sous l'angle de la morale, «c'est interroger un impensé qui, faute d'être radical, n'en est pas moins paradoxal $» .{ }^{36}$ Le recours à la morale est, quelque fois, une réponse à l'obscurité et à l'inintelligibilité de la loi. ${ }^{37}$ Le droit n'ayant de sens que s'il est compris de tous et que s'il bénéficie d'une certaine popularité. ${ }^{38} \mathrm{Il}$ importe donc de dépasser l'image du juge selon laquelle ce dernier ne serait de façon strictement absolue, que la « bouche de la loi », ${ }^{39}$ et dans le cadre de cette réflexion, que la «bouche de la Constitution " ${ }^{40}$ Pour paraphraser Lanzara dans sa thèse sur Les modes prétoriens d'aménagement des principes du droit des obligations, l'on écrira que le juge constitutionnel devra " approfondir » les principes, les «étendre », les « combler », ou encore les " ramifier », afin d' »inventer les solutions de droit $»{ }^{41}$ Cette démarche interprétative, sinon révélatrice du droit, oblige le juge parfois à convoquer les règles morales dans son office.

Cette étude aura le mérite, sur le plan théorique, d'actualiser le débat sur l'efficacité de l'immutabilité des normes de référence en matière de constitutionnalité. Elle permettra de réfléchir sur la hiérarchie des normes et la difficile garantie de l'intangibilité des limites de la Constitution. ${ }^{42}$ De plus, la contribution est une opportunité de s'interroger aussi sur le juge constitutionnel, du moins sur sa fonction, et au-delà, de s'assurer qu'il ne s'écarte pas des délimitations de son office, à savoir, l'ensemble des devoirs et des pouvoirs attachés à la fonction. ${ }^{43}$ En effet, il n'est pas rare en Afrique de constater que le juge constitutionnel ne s'est pas privé d'instrumentaliser la notion originellement coloniale et importée ${ }^{44}$ d'ordre public pour écarter certaines règles de droit traditionnel afin de préserver implicitement, en tout cas sous les apparences rassurantes de la légalité, l'idéologie officielle. Dans certains cas africains, par exemple en ayant recours de manière abusive à l'expression em-

36 BAUMERT (R.), « Une 'vision morale' de la justice constitutionnelle sous la République de Weimar », Jus Politicum, Revue internationale de droit politique, $\mathrm{n}^{\circ} 4,2010$, p. 1.

37 Or, comme le souligne MALAURIE (P.), « une loi inintelligible est une mascarade juridique » et remarque d'ailleurs que "l'intelligibilité est nécessaire à tout le droit : Constitutions, traités internationaux, décisions judiciaires, actes administratifs, actes publics et privés ».

38 MALAURIE (P.), «L'intelligibilité des lois », Pouvoirs 2005/3 (n 114), p. 131.

39 En réalité, MONTESQUIEU aurait plutôt dit « la bouche qui prononce les paroles de la loi ». De l'Esprit des lois, in Euvres de Montesquieu, éd. complète dirigée par M. Colin De PLANCY, Librairie de jurisprudence et d'Administration, 1825, p. 89.

40 La Constitution peut être du point de vue matériel et du point de vue formel.

41 LANZARA (D.), Les modes prétoriens d'aménagement des principes du droit des obligations, Thèse, Université Nice Sophia Antipolis, 2014, p. 2.

42 OUEDRAGO (M. S.), La lutte contre la fraude à la Constitution en Afrique noire francophone, Thèse, Bordeaux, 2011, p. 265.

43 CORNU (G.), Vocabulaire juridique, Paris, PUF, 2008 (1987), p. 633.

44 Paul Gérard POUGOUE parle d'ordre public d'occidentalisation, c'est-à-dire une technique commode utilisée par le juge comme instrument d'aliénation culturelle pour étouffer l'émergence d'un droit national dans Gérard Conac, « Le juge et la construction de l'État de droit en Afrique francophone », dans L'État de droit. Mélanges en l'honneur de Guy Braibant, Paris, Dalloz, 1996, p. 105 , à la page 112 . 
brouillée d' « ordre public », le juge a pu cautionner, sous les oripeaux du droit, l'autoritarisme des régimes politiques africains, comme il a eu tendance à " délégaliser » certaines matières pour affaiblir le Parlement au profit d'un pouvoir exécutif puissant ${ }^{45}$.

Sur plan théorique, cette étude constitue une opportunité de s'interroger sur l'autorité juridique des interprétations constitutionnelles. ${ }^{46}$ De plus, il s'agira de réfléchir sur le comportement du juge constitutionnel en Afrique francophone envers le respect de la loi fondamentale et de s'interroger sur les facteurs psychologiques et parfois sociologiques qui motivent la décision du juge constitutionnel. Il s'agira surtout d'actualiser le « bilan d'un demi-siècle de constitutionnalisme en Afrique $»,{ }^{47}$ constitutionnalisme pris au sens Lavroff, c'est-à-dire « la volonté de soumettre l'exercice du pouvoir à des règles écrites préétablies et déterminées en fonction d'un but choisi rationnellement $»{ }^{48}$ Enfin, il s'agira surtout de comprendre comment l'indétermination du droit conduit nécessairement le juge à faire appel à des considérations morales, politiques ou autres. ${ }^{49}$ En admettant que le juge se conforme au droit lorsque ce dernier est clair, faut-il également admettre qu'il se conforme à autre chose lorsque le droit n'est pas clair mais indéterminé? Les tenants de la thèse du " réalisme tempéré » ou du « formalisme éclairé » admettent donc implicitement que le juge fait appel à des conceptions morales ou des idéologies politiques et autres considérations extra-juridiques au sens strict du mot, considérations qu'il ne faudrait pas réduire pour autant à des choix personnels relevant de l'idiosyncrasie du juge. ${ }^{50}$

S'il est établi aujourd'hui que le juge concourt à la création du droit positif, quelle est la portée réelle du recours du juge à la règle morale? En somme, il s'agit de comprendre comment le juge constitutionnel appréhende l'incursion de la morale dans son office.

La réflexion sur la morale dans l'office du juge constitutionnel en Afrique francophone révèle surtout que l'office du juge se trouve mué et se convertit en siège de moralisation de la vie politique. Une précision des limites de l'office du juge s'impose face au dépérissement de la vie politique marquée aujourd'hui par l'implication des acteurs politiques dans

45 TOKO (P. W.), « Le juge qui crée le droit est-il un juge qui gouverne? », Les Cahiers de droit, 54 (1), 2013, p. 155.

46 LEBRUN (P. B.), « De l'autorité en droit, et des concepts qui la côtoient », Empan 2016/1 (n 101), p. 87-91.

47 KPODAR (A.), «Bilan sur un demi-siècle de constitutionnalisme en Afrique », in La Constitution béninoise du 11 décembre 1990 : un modèle pour l'Afrique? Mélanges en l'honneur de Maurice Ahanhanzo-Glélé, Paris, L’Harmattan, 2014, p. 89-126.

48 LAVROFF (G. D.), " Les tendances du constitutionnalisme africain », in Gérard CONAC (dir.), Dynamiques etfinalités des droits africains, Paris, Economica, 1980; Voir aussi BALDE (S.), La convergence des modèles constitutionnels : Etudes de cas en Afrique subsaharienne, Paris, Publibook, 2011, p. 45.

49 Idem.

50 BRUNET (P.), « Analyse réaliste du jugement juridique », Cahiers philosophiques 2016/4 ( ${ }^{\circ}$ 147), p. 17. 
les « affaires » de scandales politico-financiers. ${ }^{51} \mathrm{Si}$ Aristote avait affirmé que «la justice est un juste milieu si du moins le juge en est un ", c'est que la personne que la société place en position de juger ne cesse pas d'être un individu dès lors qu'il endosse les attributs de la fonction. ${ }^{52}$ Aucun système judiciaire, même le plus soucieux d'équité, ne peut éliminer totalement la part subjective dans une décision de justice. ${ }^{53}$ Notre analyse portera sur l'irruption évidente de la morale dans le constitutionnalisme (I), tout en montrant la dérision du contrôle de la morale constitutionnelle par le juge constitutionnel (II).

\section{L'irruption évidente de la morale dans le constitutionnalisme}

Le réalisme juridique ${ }^{54}$ entend souligner la dimension subjective qu'implique l'acte de juger et la part importante de pouvoir discrétionnaire dont bénéficient les juges. D'une part, la décision du juge constitutionnel est présentée comme un acte d'exécution ou de pure application du droit existant (de la loi ou d'un précédent) pour le seul motif que le droit existe (formalisme extrême). ${ }^{55}$ D'autre part, le juge constitutionnel crée du droit nouveau en même temps qu'il applique du droit existant car tout acte d'application d'une norme est un acte de création d'une autre norme. ${ }^{56}$ Enfin, il faut relever que le juge constitutionnel n'est

51 De MONTECLER (M.-C.), « La 'moralisation’ bute sur la suppression de la réserve parlementaire », AJDA 2017, p. 1588.

52 ASSIER-ANDRIEU (L.), " Le juge, la loi et le citoyen » in La qualité des décisions de justice, Etudes réunies par MBONGO (P.), Editions du Conseil de l'Europe, p. 13.

53 Idem.

54 La conception réaliste d'une décision de justice est d'abord une critique de la conception traditionnelle elle-même qualifiée de «formaliste », selon laquelle les juges appliquent les règles à des faits de façon à la fois délibérative, logique et mécanique, de telle sorte que le processus de décision judiciaire se réduit à un syllogisme. À cette conception, les réalistes opposent la dimension intuitive : les décisions des juges leur apparaissent motivées par une grande variété de facteurs (leurs choix politiques, leurs idées morales et/ou politiques, leurs valeurs personnelles, leurs émotions) de sorte que leur décision est d'abord le produit de ces facteurs que les raisons juridiques viendront habiller par la suite en une décision elle-même juridique. Les règles juridiques sont plutôt vues comme des classifications approximatives des décisions obtenues sur d'autres fondements, elles sont d'un usage limité dans la prédiction des décisions judiciaires. La justification juridique est donc analysée comme une rationalisation. On retrouve chez tous les auteurs réalistes l'idée que les juges peuvent toujours trouver une décision antérieure qui pourra leur servir à justifier n'importe quelle conclusion qui leur semble souhaitable, de sorte que, dans la plupart des cas, sinon tous, les juges doivent choisir entre des interprétations conflictuelles des décisions antérieures et ce choix se fait sur des fondements " extra-juridiques ». Voir aussi, TROPER (M.), " Le réalisme et le juge constitutionnel », Cahier du Conseil constitutionnel $n^{\circ} 22$ (Dossier : Le réalisme en droit constitutionnel) - juin 2007, https://www.conseil-constitutionnel.fr/nouveaux-cahiers-du-conseil-constituti onnel/le-realisme-et-le-juge-constitutionnel consulté le 1er juin 2018.

55 Cette première conception simpliste, voire grossière, a elle-même été revue et l'on a alors cherché à montrer, pour les uns, que le jugement n'est pas un acte de création ex nihilo mais, au mieux, d'interprétation car il reste toujours dépendant de la loi ou du précédent (positivisme classique).

56 KELSEN (H.), Théorie pure du droit, traduit par THEVENAZ H., Neuchâtel, Editions de la Baconnière, 1953, 205 pages. 
jamais un créateur de droit car il est toujours tenu de se référer à des standards ou des principes susceptibles de fonder plusieurs décisions futures.$^{57}$ Le juge va dans certaines circonstances convoquer diligemment dans son office la règle morale (A) mais cette convocation aura des effets controversés (B).

\section{A. La mobilisation diligente de la morale}

L'idée « idyllique » d'une jurisprudence qui appliquerait exclusivement la loi avec une volonté généreuse de collaboration avec le législateur demeure une utopie de théoriciens. Loin de dépeindre la réalité, cela va masquer, difficilement au demeurant, les figures bien distinctes que la doctrine a depuis longtemps décrites. ${ }^{58}$ Mais à l'analyse, la prise en compte de la morale par le juge est très laborieuse et révèle une certaine ambivalence dans l'office du juge constitutionnel. De même, le juge administratif va recourir aux valeurs morales avec des fortunes diverses.

1. Les occurrences de la morale dans les décisions des juridictions constitutionnelles spécialisées

Il est incontestable que le but assigné à tout « procès constitutionnel $»^{59}$ est de purger l'ordre constitutionnel des dispositions inconstitutionnelles c'est-à-dire, assurer une prééminence de la Constitution dans l'ordre interne. Aussi, le juge constitutionnel, dans sa démarche, va apprécier avant tout, la conformité des normes à la Constitution. Son repère est donc la Constitution. Le recours à la morale dans l'office du juge constitutionnel est en réalité une « exception ». L'étude de la morale dans la jurisprudence constitutionnelle révèle donc une hésitation initiale du juge constitutionnel à y recourir. Mais comme le droit n'est pas une discipline autonome et est considéré par Harnay et Marciano comme le soutien de la morale, ajoutant des sanctions temporelles aux sanctions de la conscience, ${ }^{60}$ cette hésitation sera reléguée aux " oubliettes ». Selon ces auteurs, le terme « ajouter » renforce bien l'idée que, « dans le processus d'ordonnancement des sociétés, le droit n'intervient qu'après la morale ». ${ }^{61}$ De manière évidente, si le droit est le support de la morale, le juge doit mettre son pouvoir au service de la morale. Il faut souligner que cette rupture, pragmatique, avec le positivisme, trouve ses explications dans l'analyse morale du statut du juge constitutionnel. Ainsi, la véritable question est sans doute de déterminer la marge de liberté qui peut être reconnue au juge dans son office. Pour résoudre ce problème essentiel, il faut

57 DWORKIN (R.), « Controverse constitutionnelle », Pouvoirs $n^{\circ}$ 59, 1991, p. 5.

58 MOLFESSIS (N.), « Loi et jurisprudence », Pouvoirs $n^{\circ} 126,2008$, p. 88.

59 JAN (P.), Le procès constitutionnel, LGDJ, 2010, 233 p.

60 HARNAY (S.) et MARCIANO (A.), « I. le juge, le philosophe et le scientifique. Commentaire sur the problematics of moral and legal theory de Richard A. POSNER », Revue internationale de droit économique 2001/1 (t. XV, 1), p. 104.

61 Idem. 
se référer aux différentes méthodes d'interprétation des textes et se demander d'abord si le juge doit se cantonner à des méthodes d'interprétation intrinsèque ou s'il peut s'évader vers des formes d'interprétation extrinsèque. Gény affirma alors « que les éléments purement formels et logiques... sont insuffisants à satisfaire les desideratas de la vie juridique ", si bien qu'il incombe aux juristes de rechercher "en dehors et au-dessus de ces éléments les moyens de remplir toute leur mission $\gg .{ }^{62}$

Plusieurs questions amènent le juge constitutionnel à sortir du cadre de sa « fonction originelle » ou tout au moins, à adopter une démarche l'obligeant à recourir à la règle morale. Il s'agit surtout de la bioéthique, ${ }^{63}$ de la dignité, du mariage des personnes de même sexe et des questions plus ou moins liées aux opérations électorales, ${ }^{64} \ldots$ Est-ce suffisant pour dire comme Chénedé, que le « constitutionnalisme est un jusnaturalisme »? ${ }^{65}$ En effet, selon ce courant doctrinal en théorie du droit, la validité juridique d'une règle dépend principalement de son contenu substantiel et de sa conformité à la morale, et cette moralité peut être découverte par un acte de connaissance. ${ }^{66}$ A l'évidence, la morale est présente dans la juridiction constitutionnelle, soit le juge va recourir à la règle morale dans sa mission, soit il est saisi par des questions de moralité. ${ }^{67}$

Sur la référence à la dignité par le juge constitutionnel, la question a été abordée par Saint-James dans «Réflexions sur la dignité de l'être humain en tant que concept juridique du droit français ». ${ }^{68}$ Elle reconnait l'unanimité qui est faite aujourd'hui sur l'évolution du concept de dignité. Un concept qui, curieusement, n'est pas expressément par la Constitu-

62 BERGEL (J.-L.), " L'office du juge », Actes de colloques, www.senat.fr/colloques/ office_du_juge49.htlm consulté le 27 juillet 2018.

63 En France, le juge constitutionnel a été saisi, de la loi tendant à modifier la loi n²011-814 relative à la bioéthique en autorisant sous certaines conditions la recherche sur l'embryon et les cellules souches embryonnaires.

64 La décision n 94-343 DC du 27 juillet 1994 sur « la loi relative au respect du corps humain et la loi relative au don et à l'utilisation des éléments et produits du corps humain, à l'assistance médicale à la procréation et au diagnostic prénatal ». Voir également, Du BOIS DE GAUDUSSON (J.), «Les élections à l'épreuve de l'Afrique », Cahier du Conseil constitutionnel n¹3, janvier 2003. Voir aussi MASSINA (P.), « Le juge constitutionnel africain francophone : entre politique et droit », Revue française de droit constitutionnel 2017/3, p. 641-670.

65 Petites affiches, 20/02/2013 n 37, page 6.

66 En d'autres termes, pour le jusnaturalisme, le « devoir être » du droit peut et doit être inféré de l' « être » d'un monde moral ou d'une substance ou d'un principe auquel la connaissance humaine peut accéder. LA TORRE (M.), « Le modèle hiérarchique et le Concept de droit de Hart », https:// revus.revues.org/2728, consulté le 30 juin 2017.

67 «Moralité, quand tu nous tiens ». En effet, en vertu d'une autorité sublimée par une fonction de veiller à la cohérence de l'ordre juridique assurée par la juridiction constitutionnelle, il est proposé que sa jurisprudence ait force contraignante pour que sa mission soit effective et efficace. Confère, SCHOETTL (J.-É.), « Moralité, quand tu nous tiens », Petites affiches, $2017 \mathrm{n}^{\circ}$ 118, page 7. Voir aussi DESAULNAY (O.), « L'autorité des décisions du Conseil constitutionnel vue par la Cour de cassation », Les Nouveaux Cahiers du Conseil constitutionnel 2011/1 (N 30), p. 34.

68 Recueil Dalloz 1997 p. 61. 
tion française, ${ }^{69}$ à l'inverse d'autres Constitutions. La mention figure dès le préambule de la Constitution du Togo ${ }^{70}$ et dans celui de la Constitution du Bénin. ${ }^{71}$ Jusqu'en 1994 en France, le Conseil constitutionnel ne s'y était jamais référé. ${ }^{72}$ Aussi, faut-il signaler qu'en France, le Comité consultatif pour la révision de la Constitution avait souhaité inscrire dans le texte constitutionnel le droit de chacun à la dignité de sa personne. ${ }^{73}$ Lors des débats sur le projet de loi relatif au respect du corps humain, le respect de la dignité humaine est invoqué comme l'un des objectifs du texte. ${ }^{74} \mathrm{Il}$ aurait fallu attendre la décision $\mathrm{n}^{\circ}$ 94-343 DC du 27 juillet 1994 sur « la loi relative au respect du corps humain et la loi relative au don et à l'utilisation des éléments et produits du corps humain, à l'assistance médicale à la procréation et au diagnostic prénatal », pour voir la consécration du principe de dignité. Dès lors, le principe de dignité va connaître un très grand succès puisqu'il va irriguer les différentes branches du droit ${ }^{75}$. Pour Mathieu, « fonder le principe de la sauvegarde de la dignité de la personne humaine sur la phrase préliminaire du Préambule de 1946 peut sembler quelque peu audacieux $»{ }^{76}$ En effet, la dégradation de la personne humaine invoquée dans le Préambule de 1946 renvoie nécessairement et entre autres, aux expériences « médicales » et eugéniques conduites sous l'égide de l'Allemagne nazie. ${ }^{77}$ En Afrique, le juge constitutionnel béninois dans des situations où était en cause la dignité humaine; $n$ 'a pas fait recours à ce concept, quand bien-même celui-ci figure dans le Préambule de la Constitution de 1990.

69 Il n'empêche que la notion de « dignité humaine » n'était pas, à l'époque, inconnue du droit français puisque sa première apparition date, semble-t-il, de la loi n ${ }^{\circ} 86-1067$ du 30 septembre 1986, modifiée, relative à la liberté de la communication.

70 «...un Etat de Droit dans lequel les droits fondamentaux de l'Homme, les libertés publiques et la dignité de la personne humaine doivent être garantis et protégés », Voir le Préambule de la Constitution du 14 octobre 1992 modifiée le 31 décembre 2002 et à l'article 11 de la Constitution du Togo : « Tous les êtres humains sont égaux en dignité et en droit».

71 Constitution de la République du Bénin du 11 décembre 1990.

72 MATHIEU (B.), «Bioéthique : un juge constitutionnel réservé face aux défis de la science », $R F$ DA, 1994, p. 1019.

73 Rapport publié au JO 16 févr. 1993, p. 2547. Voir aussi, PETCH T., «La dignité humaine. Du droit à l'éthique de la relation », Revue internationale d'éthique sociale et gouvernementale, vol. 3 , $\mathrm{n}^{\circ} 2,2001$.

74 CABANEL (G.), Rapport au nom de la Commission des lois du Sénat, n² 230-1993-1994 et déclaration du garde des Sceaux, débats Ass. nat., 7 avr. 1994, J.O. p. 633.

75 Décision $n^{\circ}$ 94-343/344 DC du 27 juillet 1994 sur la Loi relative au respect du corps humain et loi relative au don et à l'utilisation des éléments et produits du corps humain, à l'assistance médicale à la procréation et au diagnostic prénatal, précitée.

76 « Considérant que le Préambule de la Constitution de 1946 a réaffirmé et proclamé des droits, libertés et principes constitutionnels en soulignant d'emblée que : "Au lendemain de la victoire remportée par les peuples libres sur les régimes qui ont tenté d'asservir et de dégrader la personne humaine, le peuple français proclame à nouveau que tout être humain, sans distinction de race, de religion ni de croyance, possède des droits inaliénables et sacrés"; qu'il en ressort que la sauvegarde de la dignité de la personne humaine contre toute forme d'asservissement et de dégradation est un principe à valeur constitutionnelle ».

77 MATHIEU (B.), précité. 
Le juge constitutionnel béninois condamne «l'usage de la contrainte, des pressions et tous actes de violence caractérisés, provoquant chez la victime des souffrances physiques ou mentales, ou aboutissant à une désintégration de sa personnalité ou l'anéantissement de sa volonté $» .{ }^{78}$ Une condamnation fidèle à sa politique de protection de l'intégrité humaine. Pour Hounaké, la spécificité des droits découlant de la protection de l'intégrité de la personne humaine, leur permet d'échapper à toute considération de proportionnalité. Il précise que ce sont des « droits dits inconditionnels de portée absolue ». ${ }^{79}$

En effet, pour que le comportement en cause rentre dans la catégorie des traitements condamnables, il faut prendre en compte une certaine intensité. ${ }^{80}$ Bioy écrit à propos que " on touche ici au cour de la dignité : par la manipulation du corps ou par celle de l'esprit, l'individu peut être rabaissé au rang de chose aux mains d'autrui $\gg .{ }^{81}$ Le juge constitutionnel béninois retient de son côté que "les traitements cruels inhumains et dégradants s'apprécient non seulement en fonction de leur effet sur l'état physique ou mental de l'individu mais également au regard de leur durée, de leur caractère délibéré et des circonstances dans lesquelles ils ont été infligés $»{ }^{82}$

De même, la morale s'invite aussi en matière électorale. Parent constate que les magistrats ne se privent pas de commenter les mauvaises pratiques des candidats aux élections. Et il est de plus en plus courant que les juges recourent à cette fin à des notions dont la connotation morale est évidente. Au concept de sincérité se sont ajoutées d'autres notions. Que l'on songe au Conseil constitutionnel en France, qui, en 2002, annulait les opérations électorales d'une petite commune de l'Aude ${ }^{83}$ au motif que l'implication du maire dans l'organisation la consultation du second tour des présidentielles, a révélé des procédés " incompatibles avec la dignité du scrutin $»{ }^{84}$ Mais la morale s'invite dans d'autres domaines autre celui des élections.

En France, en 2016, saisi sur les questions de la suppression du délai de réflexion avant un acte chirurgical et la recherche sur l'embryon, le Conseil constitutionnel a considéré que la demande d'IVG et la confirmation de l'IVG ne pouvant avoir lieu le même jour, la suppression du délai de réflexion n'était pas contraire à « l'équilibre » de la loi VEIL. D’autre part, répondant à ceux qui estimaient que tout achat ou a fortiori les actes médicaux pouvaient donner lieu à un délai de rétractation, il a mentionné qu'aucune disposition constitutionnelle n'imposant « de façon générale » de délai de réflexion avant un acte chirurgical, la

78 DCC 98-065 du 05 Août 1998, Recueil 1998, p. 321.

79 HOUNAKE (K)., « L'exigence de proportionnalité dans la jurisprudence de la Cour constitutionnelle du Bénin », RTSJ, janvier 2015, p. 166.

80 BADET (G.), Les attributions originales de la Cour constitutionnelle du Bénin, FES, 2013, p. 129.

81 BIOY (X.), Droits fondamentaux et libertés publiques, Paris, Montchrestien, 2011 p. 391.

82 DCC 99-011 du 4 février 1999, Recueil 1999, p. 35 et suivantes.

83 Cons. const., 8 mai 2002, Décision portant proclamation des élections présidentielles, Recueil, p. 114.

$84 R D P, 2011, \mathrm{n}^{\circ} 5$, p. 1213. 
suppression du délai de réflexion est conforme à la Constitution. Enfin, le Conseil constitutionnel autorise la recherche sur l'embryon. ${ }^{85}$

La complexité du recours à la morale par le juge constitutionnel invite à examiner comment la règle morale motive les jurisprudences ordinaires.

2. Les juridictions ordinaires confrontées aux questions de constitutionnalité et le recours à la morale

Généralement, les juridictions ordinaires sont confrontées à des questions de constitutionnalité. Le cas échéant, elles sont tenues de soulever une question d'inconstitutionnalité 86 lorsqu'elles considèrent qu'une norme ayant force de loi s'avérant applicable à la cause dont elles connaissent, dont la validité est de nature à déterminer le sens de leur décision, peut aller à l'encontre de la Constitution. Le facteur déterminant pour soulever une question d'inconstitutionnalité est donc le doute du juge ou du tribunal. Selon Hounaké, sur la question d'exception d'inconstitutionnalité : "le système constitutionnel des pays africains francophones fut jusqu'au $1^{\text {er }}$ mars $2010^{87}$, plus avancé que celui de la France ${ }^{88}$. En effet,

85 Le Conseil constitutionnel a estimé que « le paragraphe III de l'article 155 qui permet, dans le cadre de l'assistance médicale à la procréation et avec le consentement des membres du couple, la réalisation de recherches biomédicales sur des gamètes destinés à constituer un embryon ou sur un embryon in vitro avant ou après son transfert à des fins de gestation "était conforme à la Constitution ». Voir, http://www.genethique.org/fr/le-conseil-constitutionnel-valide-le-naufrage-bi oethique-de-la-loi-sante-64798.html\#.WmmlAHnLjIU consulté le 27 juin 2017.

86 La différence fondamentale entre l'exception d'inconstitutionnalité et la technique dite de question préjudicielle réside au niveau de la possibilité reconnue ou non au juge devant qui la question est posée d'apprécier ou non son bien-fondé. Selon le Professeur Guillaume Drago est qualifiée d'exception d'inconstitutionnalité la technique par laquelle le juge saisi d'une affaire peut lui-même répondre à la question et trancher le contentieux de constitutionnalité voir en ce sens, DRAGO (G.), «Exception d'inconstitutionnalité : Prolégomènes d'une pratique contentieuse », JCP/La Semaine Juridique-Edition générale, numéro 49.3 décembre 2008, p. 14. Cette définition rejoint celle de Georges Burdeau pour qui l'exception d'inconstitutionnalité consiste pour « un particulier menacé de sanction pour avoir violé une loi demande à la juridiction devant laquelle il est poursuivi de constater l'inconstitutionnalité de cette loi et, partant, d'écarter son application (BURDEAU G.) «Le statut du pouvoir » Traité de science politique, T. IV, $3^{\mathrm{e}}$ éd., LGDJ, 1984, p. 434). En revanche, dans le cas de la question préjudicielle de constitutionnalité, les juges ordinaires-privés de la capacité de juger eux-mêmes la loi-doivent renvoyer la question de constitutionnalité au juge constitutionnel ». V. sur ce point FAVOREU (L.), « La question préjudicielle de constitutionnalité : Retour sur un débat récurrent », in Droit et politique à la croisée des cultures, Mél., Philippe Ardant, LGDJ, 1999, p.265-273, spéc., p. 266.

87 C'est à cette date que la question prioritaire de constitutionnalité introduite dans la Constitution française par la Loi constitutionnelle n'2008-724 du 23 juillet 2008 (JORF, du 24 juillet 2008, p. 11890) est devenue effective.

88 En France, l'expression consacrée est la question prioritaire de constitutionnalité. Une autre spécificité du système français est que la question posée n'est pas tranchée (sauf exceptions) par le premier juge saisi. Celui-ci doit envoyer la résolution de la question à la juridiction suprême de son ordre. V. DE VILLIERS (M.) et LE DIVELLEC (A.), Dictionnaire du droit constitutionnel, $8^{\mathrm{e}}$ éd., Paris, Sirey, 2011, p. 298-299. 
l'exception d'inconstitutionnalité a été introduite dans les dispositions fondamentales des Etats africains dès les années 1990 avec la troisième vague du constitutionnalisme ${ }^{89} »{ }^{90}$ Sous une autre perspective, la question d'inconstitutionnalité doit impérativement être soulevée lorsque l'alternative est l'inapplication d'une norme ayant valeur de loi. Le doute de constitutionnalité ne peut en aucun cas être résolu par l'inapplication de la loi, mais par la mise en cause de sa validité devant le juge constitutionnel.

Le recours à la morale par le juge ordinaire a été traité très tôt par le doyen Hauriou. ${ }^{91}$ Pour le maître de « l'Ecole de Toulouse », le juge administratif s'adonne à un " contrôle de moralité » lorsqu'il est saisi d'un recours pour excès de pouvoir fondé sur le détournement de pouvoir. Le détournement de pouvoir étant l'utilisation d'une compétence dans un but non prévu par les textes légaux ou pour favoriser des desseins personnels. Le problème fondamental est celui de la preuve du détournement de pouvoir. L'expression « contrôle de moralité » a été reprise par Welter dans sa thèse intitulée : "Le contrôle juridictionnel de la moralité administrative $"{ }^{92}$ Il s'agit pour le juge de l'administration d'avoir une appréhension objective d'une donnée subjective. ${ }^{93}$ L'appréciation de cette dernière se heurte au pragmatisme procédural, au point de poser la question de la dépendance du détournement de pouvoir par rapport à la morale et sa propension à porter un jugement de valeur sur l'élément intentionnel de l'acte. ${ }^{94}$

89 Dans son célèbre article « Les nouvelles tendances du constitutionnalisme africain », le professeur AHADZI-NONOU a distingué dans l'évolution constitutionnelle de ces pays trois cycles constitutionnels. Le premier va des années 1958-59 à 1964-1965 et correspond à l'accélération du mouvement de décolonisation et à l'accession à l'indépendance de la plupart des pays africains. Le second a débuté aux alentours de 1964-1965 et a couru jusqu'à 1990. Le troisième a commencé en 1990 avec la chute des dictatures et l'avènement de la démocratie. Les nouvelles tendances du constitutionnalisme africain op. cit., p. 5-8.

90 Dans certains pays, comme le Togo, la question peut être soulevée devant les cours et tribunaux. Il en est de même au Niger où "l'inconstitutionnalité peut être soulevée devant toute juridiction ". De même, au Bénin, l'exception d'inconstitutionnalité peut être invoquée par tout citoyen "dans une affaire qui le concerne devant une juridiction ». En revanche, au Sénégal, cette voie de recours est limitée dans sa mise en œuvre. L'exception ne peut être soulevée que devant le Conseil d'Etat et la Cour de cassation. Cette restriction permet ainsi de filtrer les recours par voie d'exception qui sont de ce fait, irrecevables devant les juridictions d'instance et d'appel.

91 HAURIOU (M.), Droit administratif, Rép. Becquet, t. 14, 1897.

92 Thèse de doctorat soutenue à Nancy en 1929. La problématique est ancrée sur le contrôle juridictionnel de la moralité administrative tend à faire prendre à I' administration conscience d'ellemême, notamment en ce qu'il vise à la débarrasser de certaines influences politiques néfastes qui, dans le régime démocratique, troublent trop souvent son fonctionnement et la détournent de sa tâche, qui consiste à procéder à un règlement impartial, - sine ira et studio, selon le mot du Latin -, des questions intéressant l'ensemble du public.

93 HUMMEL (J.), « La théorie de la moralité administrative et l'erreur manifeste d'appréciation », La Revue administrative, $\mathrm{n}^{\circ} 291,1996$, p. 335.

94 BALLANDRAS-ROZET (C.), " Réflexions sur la dimension morale du détournement de pouvoir », AJDA, 2007 p. 2236. 
Quand l'on envisage d'intégrer la fonction publique, c'est que l'on a accepté intrinsèquement de se soumettre à cette « culture du devoir ${ }^{95}$ qu'impose toute sélection méritocratique. La culture de l'action et du service publics est vitale à une éthique minimale du fonctionnaire républicain. Mais alors quelles sont les principaux traits de cette éthique ou, en d'autres termes, quelles sont les valeurs auxquelles se réfère, instance après instance, la jurisprudence ${ }^{96}$ En réalité, réunir les conditions exigées par le statut n'est pas suffisant. Les candidats à l'emploi public doivent répondre non seulement aux conditions générales et spéciales d'aptitude requises par les textes, mais ils doivent aussi répondre aux « garanties de moralité désirables $\gg .{ }^{97}$

Il s'agit là d'une exigence justifiée par l'intérêt du service que le juge retient comme principe normatif, même sans texte. Aujourd'hui, la légalité ne peut être réduite à son sens strict, les sources écrites seules ne suffisent plus pour évoquer ce principe. En effet, la jurisprudence peut y ajouter une orientation générale fortement corrélée au bien commun. ${ }^{98} \mathrm{La}$ preuve en est que la condition de moralité s'impose non seulement aux fonctionnaires mais aussi aux candidats à un emploi d'agent contractuel. ${ }^{99}$ Le juge prend en compte également la moralité du candidat à l'accès à la fonction publique. ${ }^{100} \mathrm{La}$ jurisprudence est abondante en la matière. ${ }^{101}$ Ainsi, le juge a-t-il pu accepter que l'autorité administrative refuse d'admettre une personne à se présenter au concours de commissaire de police, en se fondant à juste titre sur un comportement qui avait signalé l'intéressé à l'attention des services de police, notamment, pour conduite en état d'ivresse.

La question du recours à la morale par le juge est très présente devant le juge pénal : l'intime conviction du juge et du juré. Il ne faut pas l'assimiler à une impression, mais elle exige de passer au crible de la raison toutes les composantes du dossier, chaque élément de preuve, chaque moyen de défense. Il s'agit donc d'une méthode de travail qui exclut toute attitude superficielle. Cela requiert une rigueur dans la réflexion, dans le raisonnement, une

95 L'expression est de LIPOVETSKY (G.) dans Le crépuscule du devoir, N.R.F., Essais, Galimard, 1992.

96 MALLOL (F.), « Condition de moralité et accès a la fonction publique », Petites affiches, 1995, n $\circ 75$, p. 4.

97 C.E., 5 juillet 1851, Rouget, Rec., p. 498.

98 MALLOL (F.), op. cit.

99 C.E., 22 novembre 1961, Wolff, Tables, p. 1082; C.E., 26 mai 1976, ministre d'Etat, ministre de l'Intérieur c/ Sieur Jacqmin, AJDA 1976, p. 630.

100 Cette appréciation est très subjective, probablement la plus subjective, car, liée au comportement des candidats à la fonction publique dans leur vie privée.

101 C.E., 11 décembre 1987, Ministre de l'Intérieur c/ Paterna, Tables, p. 778. La sévérité du juge s'explique en l'espèce par le niveau de l'emploi sollicité. Par contre, le candidat à un concours de surveillant de l'administration pénitentiaire ne peut être évincé pour des «faits d'éthylisme », eu égard à leur ancienneté et à la circonstance que l'intéressé s'était depuis lors amendé : C.E., 10 juin 1991, Garde des sceaux, ministre de la Justice c/ M. Vizier, AJDA 1991, p. 504, chron. C. Maugüe et R. Schwartz. 
forme d'éthique et d'humilité devant la science, devant les paroles, avant de décider. ${ }^{102}$ Beccaria, ${ }^{103}$ le « père du droit pénal moderne », dans le Traité des Délits et des Peines, qualifiait cette intime conviction de preuve morale qu'il oppose aux preuves légales. Aujourd'hui, il faut souligner que, l'immixtion de la valeur morale via la seule prise en compte de l'intime conviction, est consacrée par la juridiction constitutionnelle en France. ${ }^{104}$ La juridiction constitutionnelle précise que «le grief tiré de ce que les dispositions critiquées laisseraient à cette juridiction un pouvoir arbitraire pour décider de la culpabilité d'un accusé doit être écarté ». Bachelet O., s'est, à cette occasion, demandé s'il n’y a pas de paradoxe et de divination dans la motivation des verdicts d'assise. Ainsi, écrira-t-il, qu' " à l'aune d'une conception divinatoire de l'intime conviction, le Conseil (constitutionnel) en vient à accepter le paradoxe consistant à n'exiger en matière criminelle qu'une motivation elliptique... ». ${ }^{105}$

Enfin, le régime juridique applicable aux entreprises en difficulté instauré par l'OHADA, prévoit une procédure qui est, pendant toute sa durée sous surveillance constante. Celle du conciliateur en premier lieu, dont le professionnalisme et la moralité ${ }^{106}$ tels qu'exigés par l'Acte uniforme, garantissent le sérieux de cette surveillance; celle du juge ensuite, qui traitera les informations transmises par le conciliateur dans le sens de la plus grande rigueur. ${ }^{107}$

102 FAYOL-NOIRETERRE (J.-M.), « L'intime conviction, fondement de l'acte de juger », Informations sociales $2005 / 7, \mathrm{n}^{\circ} 127$, p. $46-47$.

103 Cesare BECCARIA, marquis de Gualdrasco et Villareggio. Il est né le 15 mars 1738 à Milan où il est mort le 28 novembre 1794. Il est un juriste, criminaliste, philosophe, économiste et homme de lettres italien rattaché au courant des Lumières. Dans Des délits et des peines, il fonde le droit pénal moderne et se signale notamment en développant la toute première argumentation contre la peine de mort.

104 Conseil constitutionnel, $1^{\text {er }}$ avr. 2011, $\mathrm{n}^{\circ} 2011-113 / 115$.

105 BACHELET (O.), « Motivation des verdicts d'assises : paradoxe et divination », Gazette du Palais, 2011, n 95 , p. 19.

106 Voir Article 5-4 de 1' ACTE UNIFORME PORTANT ORGANISATION DES PROCÉDURES COLLECTIVES D'APUREMENT DU PASSIF : « Dans la décision d'ouverture, le président de la juridiction compétente désigne un conciliateur. Le conciliateur doit avoir le plein exercice de ses droits civils, justifier de sa compétence professionnelle et demeurer indépendant et impartial vis-à-vis des parties concernées par la conciliation. En particulier, il ne doit pas avoir perçu, à quelque titre que ce soit, directement ou indirectement, une rémunération ou un paiement de la part du débiteur intéressé, de tout créancier du débiteur ou d'une personne qui en détient le contrôle ou est contrôlée par lui, au cours des vingt-quatre (24) mois précédant la décision d'ouverture. Aucun parent ou allié du débiteur, jusqu'au quatrième degré inclusivement, ne peut être désigné en qualité de conciliateur. Il en va de même pour tout magistrat en fonction ou ayant quitté ses fonctions depuis moins de cinq (5) ans. Dès qu'il est informé de sa désignation, le conciliateur atteste qu'il remplit, à sa connaissance, les conditions énoncées ci-dessus. Ả tout moment, durant le déroulement de la conciliation, s'il lui apparaît qu'il ne remplit plus ces conditions, il en informe sans délai le président de la juridiction compétente qui, s'il y a lieu, peut mettre fin à sa mission et nommer un remplaçant... ».

107 REILLE (F.), «L'instauration d'une conciliation à la française en Droit des entreprises en difficulté OHADA », Petites affiches, 2016, n 49, p. 7. 
En somme, le recours à la règle morale dans la prise des décisions de justice est très controversé.

\section{B. La mobilisation controversée de la morale}

Le silence du législateur consacre les prérogatives normatives du juge. Situation presque identique dans tout système juridique, le juge est contraint, pour éviter le déni de justice, de procéder au comblement des lacunes existantes malgré l'interdiction qui lui est faite de se prononcer par voie de disposition générale sur les causes qui lui sont soumises. ${ }^{108}$ En réalité, le silence ou la lacune du droit ne dispense jamais le juge de sa mission de juger. Et rien ne lui interdit d'y pallier en recourant à la règle morale. C'est dire que Dworkin a sans doute raison quand il considère que le droit n'est pas réductible aux seules règles formelles édictées par le législateur, ${ }^{109}$ mais se loge aussi, et surtout, dans les principes moraux opposables à l'État. En réalité, la question est de savoir comment le juge doit-il éviter le déni de justice tout en respectant son serment.

\section{Un évitement du risque potentiel du déni de justice}

L'attitude du juge face au silence du droit est dominée par un principe fondamental qui s'impose à tout juge et qui est inscrit depuis 1804 à l'article 4 du Code civil : "Le juge qui refusera de juger sous prétexte du silence, de l'obscurité ou de l'insuffisance de la loi, pourra être poursuivi comme coupable de déni de justice ". Le concept de déni de justice, au sens de cet article, a pour cible le fonctionnement de la jurisdictio signifie non seulement le refus de répondre aux requêtes, mais également de négligence à juger les affaires en état de l'être. Il faut préciser que le déni de justice peut résulter aussi, non pas de la carence de la juridiction saisie à fonctionner, mais de l'organisation même du service public de la justice. ${ }^{110}$

En droit français, ces hypothèses de déni de justice ont été neutralisées par le législateur qui a prévu des techniques ${ }^{111}$ de prévention des conflits négatifs de compétence, au cas où les deux ordres saisis s'estiment incompétents, et la résolution des conflits de décisions au fond, au cas où les deux ordres saisis se sont estimés compétents et ont donné une solution

108 RICOU (B.), « Le silence au service de la production juridictionnelle du droit : méthodes et politiques des juridictions ordinaires françaises », Les Cahiers de droit 563-4 (2015).

109 ALLARD (J.), « Ronald Dworkin ou le roman du droit », http://www.laviedesidees.fr/IMG/pdf/2 0140107_dworkin-2.pdf, consulté le 26 juin 2017.

110 DEGUERGUE (M.), « Les dysfonctionnements du service public de la justice », Revue française d'administration publique 2008/1 ( $\left.\mathrm{n}^{\circ} 125\right)$, p. 151-167.

111 Ces techniques, qui font intervenir le Tribunal des conflits, ont prouvé leur efficacité et les dénis de justice sous cette acception sont quasiment en voie d'extinction. 
différente. ${ }^{112}$ Toutefois, le déni de justice connaît une cure de jouvence depuis que les juridictions judiciaires recourent à cette notion pour qualifier le retard à juger. ${ }^{113}$

Ainsi, un délai de règlement excessivement long dans un litige exigeant une solution rapide « est [-il] révélateur d'un fonctionnement défectueux du service de la justice et équivaut [-il] à un déni de justice en ce qu'il prive le justiciable de la protection juridictionnelle qu'il revient à l'État de lui assurer $»,{ }^{114}$ ce qu'évoque Hounaké en des termes très imagés de «L'application à géométrie variable du déraisonnable-anormal ». ${ }^{115}$

Aristote écrit déjà au IVème siècle avant Jésus-Christ, que : «l'action injuste comporte deux extrêmes : l'un d'eux, le moindre, consiste à subir l'injustice; l'autre, le plus grave, à la commettre ». ${ }^{116}$ Comme l'a souligné Viala, l'insignifiance normative d'un texte ne saurait servir de prétexte au juge pour se dérober à cette obligation d'être libre et d'assumer souverainement son office. Pour Viala, «tout se passe comme si cette disposition législative, caractéristique de la tradition continentale de la justice, reposait sur une certaine forme d'existentialisme juridique : la relativité des valeurs plonge l'autorité dans un néant qu'il lui appartient de combler librement $» .{ }^{117}$ L'autorité judiciaire n'a pas le droit de dire « je ne sais pas », car le néant n'offrant rien qui puisse être objectivement connaissable, l'autorité judiciaire ne saurait invoquer une quelconque obscurité de la loi pour éluder son devoir de juger. ${ }^{118}$

En droit public, Favoreu considère que la notion de " déni de justice » est de ces notions vagues qui font passer le frisson sur l'échine des juristes sensibles. Pour l'auteur, la notion évoque le spectacle du plaideur importuné devant la porte close du prétoire; l'évocation est plus pénible encore s'il s'agit de droit public : c'est celle de l'administré persécuté par un potentat insensible et incompréhensif en qui se résume communément la puissance publique. ${ }^{119}$

L'interdiction du déni de justice n'est pas abordée expressément dans les lois fondamentales en vigueur dans les Etats africains. On note cependant la volonté affichée du

112 Décret $\mathrm{n}^{\circ}$ 60-728 du 25 juillet 1960 tendant à prévenir les conflits négatifs et loi du 20 avril 1932, dont le vote a été déterminé par l'affaire Rosay, qui donne au Tribunal des conflits la compétence pour trancher au fond les contrariétés de décisions définitives rendues par les deux ordres de juridiction.

113 Or, le retard à juger devant les tribunaux judiciaires est qualifié de déni de justice au sens du code de l'organisation judiciaire et entraîne l'engagement de la responsabilité de l'État.

114 CA Paris, 10 novembre 1999, D., 2000, IR, p. 31.

115 HOUNAKE (K.), op. cit., p. 159.

116 ARISTOTE, Ethique de Nicomaque, liv.V, chap.V, trad. J. VOILQUIN, Paris, Garnier-Flammarion, 1965 , p. 150.

117 « Table ronde », RDP, 2014, n 4, p. 863.

118 Idem.

119 FAVOREU (L.), « Du déni de justice en droit public français ». In Revue internationale de droit comparé. Vol. 17 N², Avril-juin 1965, p. 516-519. 
constituant de mettre fin à l'injustice qui avait prévalu avant les années $1990 .{ }^{120}$ L'existence d'un pouvoir judiciaire, auquel le plus souvent tout un titre de la Constitution y est consacré, ${ }^{121}$ illustre à suffisance cette détermination. Par ailleurs, certaines dispositions constitutionnelles expriment indirectement l'obligation au juge de dire le droit et l'interdiction de se dérober sous les lacunes pour commettre le déni. «Toute personne a droit en toute matière à ce que sa cause soit entendue et tranchée équitablement dans un délai raisonnable par une juridiction indépendante et impartiale $» .^{122}$ De même, on peut lire à l'article 9 alinéa 3 de la Constitution du Sénégal ${ }^{123}$ que « tout citoyen a droit à un double degré de juridiction dans les conditions fixées par la loi».

En évitant le déni de justice par le recours à la règle morale dans son office, le juge consacre ainsi le droit constitutionnel au recours juridictionnel effectif, c'est à dire la faculté réelle d'agir en justice, afin de demander à un juge de statuer sur le fond d'un contentieux. Le rôle du juge dans la réalisation de cette composante essentielle d'un État de droit est important. En effet, que ce soit dans son acception formelle, consistant à garantir le respect de la hiérarchie des normes, ou dans sa conception substantielle, permettant la protection des droits et libertés, le juge est très sollicité. Ainsi, se référant à la définition du déni de justice proposée par le doyen Favoreu, l'État est débiteur d'un devoir de protection juridictionnelle à l'égard des citoyens, dont le fondement réside dans le droit au juge. ${ }^{124}$

\section{Une opportunité d'évaluation du serment du juge constitutionnel}

La réflexion sur la morale dans l'office du juge, offre l'opportunité d'apprécier le serment fait par le juge au moment de son intégration dans le corps de la magistrature. ${ }^{125}$ Le juge, le plus souvent, lorsqu'il est admis à officier doit prêter serment. En référence à des exemples

120 L'Article 21 de la Constitution au Togo énonce : « La personne humaine est sacrée et inviolable. Nul ne peut être soumis à la torture ou à d'autres formes de traitements cruels, inhumains ou dégradants. Nul ne peut se soustraire à la peine encourue du fait de ces violations en invoquant l'ordre d'un supérieur ou d'une autorité publique. Tout individu, tout agent de l'Etat coupable de tels actes, soit de sa propre initiative, soit sur instruction, sera puni conformément à la loi. Tout individu, tout agent de l'Etat est délié du devoir d'obéissance lorsque l'ordre reçu constitue une atteinte grave manifeste au respect des Droits de l'Homme et des libertés publiques ». Ces dispositions témoignent de la détermination du constituant à mettre fin à l'injustice et à l'arbitraire. Voir aussi les articles 17, 18, 19, et 20 de la Constitution béninoise du 11 décembre 1990.

121 Voir Titre VI de Loi N ${ }^{\circ}$ 90-32 du 11 Décembre 1990 portant Constitution de la République du Bénin, les articles 125 et suivants.

122 Voir, article 19 alinéa de la Constitution du Togo précitée.

123 Constitution adoptée le 7 janvier 2001.

124 FAVOREU (L.), « Résurgence de la notion de déni de justice et droit au juge », Gouverner, administrer, juger, Liber amicorum Jean Waline, Dalloz, 2002, p. 513. Voir également AIVO (F. J.), Le juge constitutionnel et l'Etat de droit en Afrique : l'exemple du modèle béninois, Paris, Harmattan, 2006, $222 \mathrm{p}$.

125 Comment le juge constitutionnel peut-il recourir à la règle morale sans que sa propre moralité n'influence sa décision? À la suite de HOLMES, HUTCHESON entend la débarrasser de ses 
fournis par l'histoire constitutionnelle et par le droit comparé, une prestation de serment prend la forme d'un engagement verbal à remplir ses fonctions avec zèle et à respecter les obligations qui lui sont liées. Il s'agit donc d'un engagement « promissoire » par lequel l'intéressé fait une promesse de comportement, plutôt qu'un engagement « assertoire » par lequel il promet de dire la vérité à l'instar du serment prêté par les témoins devant les diverses juridictions. Le plus souvent, l'on admet que l'intérêt du serment consiste dans la conscience du jureur de s'exposer à un châtiment nécessaire et imparable en cas de parjure. ${ }^{126}$ Un serment est donc une promesse pour l'avenir ou l'assurance d'une vérité présente ou passée; cette promesse ou cette assurance faite en face du peuple (ou Dieu), qu'on appelle comme témoin et comme garant. ${ }^{127}$ Le juge s'engage à exercer fidèlement sa fonction. Il s'engage à respecter la règle de droit mais aussi les règles morales lorsqu'il doit interpréter les textes. ${ }^{128}$

Au Togo, aux termes de l'article 3 de la loi n ${ }^{\circ} 2004-004$ du $1^{\text {er }}$ mars 2004 sur la Cour constitutionnelle, avant d'entrer en fonction, les membres de la Cour constitutionnelle prêtent serment au cours d'une cérémonie solennelle d'installation devant le Président de la République, en présence du Président de l'Assemblée nationale et du Président du Sénat dans les termes suivants : "Je jure de bien et fidèlement accomplir mes fonctions en toute impartialité dans le respect de la constitution, de garder le secret des votes et des délibérations, de ne prendre aucune consultation sur les questions relevant de la compétence de la Cour Constitutionnelle ». De même, aux termes de l'article 7 de la loi organique ${ }^{\circ} 91-11$ du 21 août 1996 fixant statut des magistrats au Togo, le nouveau magistrat prête serment avant son entrée en fonction en ces termes : «Je jure de bien et fidèlement remplir mes fonctions, de les exercer en toute impartialité, de garder religieusement le secret des délibérations et de me conduire, en tout, comme un digne et loyal magistrat ».

connotations morales, au sens d'un «Bien » et d'un « Mal » absolus et éternels. Mais l'historicisation de la justice ne signifie pas sa dissolution : elle consiste essentiellement pour lui en un savant équilibre entre les intérêts en conflit, entre droits individuels et devoirs sociaux, tensions sans cesse renaissantes dont il fait le cœur des problèmes que le juge a à résoudre. "Lawyer's Law, and the Little, Small Dice », Tulane Law Review, vol. VII, n 1, déc. 1932.

126 SAUVAGEOT (F.), « Le serment des hautes autorités étatiques : une institution à développer? », Revue du droit public $2006-\mathrm{n}^{\circ}$ 1, page 201.

127 Le serment était un acte sacré et les conditions de son accomplissement le faisaient échapper à la fois à la banalité du quotidien et au commun des hommes. Cependant, il ne relevait pas exactement du rituel car le ritualisme religieux, au sens que lui donnait Max Weber, suppose la quotidienneté des actes accomplis (entendons leur réitération selon un code spécifique) et la dispense de la grâce. Voir LEMESLE (B.), « Le serment promis. Le serment judiciaire à partir de quelques documents angevins des XIème et XIIème siècles », Crime, Histoire \& Sociétés / Crime, History \& Societies [En ligne], Vol. 6, n² | 2002, mis en ligne le 25 février 2009, consulté le 02 octobre 2016. URL : http://chs.revues.org/410.

128 En effet, dès son entrée en fonction, le magistrat doit prêter serment de bien et fidèlement remplir ses fonctions, de garder religieusement le secret des délibérations et de se conduire en tout comme un digne et loyal magistrat. Voir, DOKHAN (D.), « Le Conseil d'Etat, garant de la déontologie des magistrats de l'ordre judiciaire », RFDA 2002, p. 768. 
"C'est dans le serment, que gît la principale cérémonie de la réception : et c'est ce serment qui attribue et accomplit en l'officier, l'ordre, le grade et, s'il faut parler ainsi, le caractère de son office et qui lui défère la puissance publique ». ${ }^{129}$

Le serment est une vieille tradition qui remonte à des siècles dans le temps. "Levo manum meam ad Dominum Deum excelsum possessorem coeli et terrae », ${ }^{130}$ disait le patriarche Abraham en prenant Dieu à témoin qu'il n'enlèverait rien au roi de Sodome, et il employait ainsi la formule de serment la plus simple et la plus exacte. ${ }^{131}$

Comme le souligne Tardif de Moidrey L., il n'y a pas de sujets aussi subtils susceptibles de fixer simultanément l'attention du philosophe et du jurisconsulte que le serment. ${ }^{132}$ En effet pour l'auteur, par sa raison d'être, sa nature et son origine, il pourrait fournir l'objet d'un traité complet, et celui qui voudrait l'entreprendre devrait aborder les plus hautes questions de la métaphysique, de la théologie et de l'histoire, car le serment est plus qu'un acte juridique, ou qu'une formalité ordinaire: c'est en quelque sorte, une grande institution: institution de droit naturel et social, se retrouvant partout et dans tous les temps, découlant de la nature même de l'homme et répondant à ses plus impérieuses exigences, corrélative à l'idée de Dieu et basée sur elle; il faudrait suivre le serment aux diverses époques de la vie des sociétés. ${ }^{133}$ Le serment est pour l'homme qui croit en Dieu, et la presque totalité des hommes y croit, ce que la parole d'honneur est pour l'homme d'honneur, ce qu'une simple déclaration est pour le véritablement honnête homme. Celui qui trompe dans une simple déclaration, ou dans une simple promesse, renonce à la confiance et au titre d'homme vrai. ${ }^{134}$ Aussi, dans le cas du serment judiciaire le juge qui viole les engagements qu'il a contractés par serment, est toujours regardé avec exécration et parfois avec mépris. ${ }^{135} \mathrm{C}$ 'est dire que normalement, le serment prêté par les juges pourrait participer à l'affermissement de leur impartialité, une garantie de la qualité de justice. Il s'agit donc d'une exigence essentielle. ${ }^{136}$ Une « mystique de l'impartialité », conduirait à tous les malentendus. ${ }^{137}$ La notion d'impartialité est à distinguer de l'indépendance des juges. Est indépendant, le juge qui ne

129 LOYSEAU, liv. 1 des offices.

130 « Je lève la main et je jure par le Seigneur le Dieu très-haut, possesseur du ciel et de la terre » (Genèse XIV, 22).

131 Genèse, ch. 14.

132 Serment litis-décision en droit français et en droit romain, Librairie de l'Académie impériale, 1860 , p. 1

133 Idem.

134 Du serment. Différence entre le serment politique et le serment judiciaire. Source gallica.bnf.fr / Bibliothèque nationale de France, consulté le 13 juillet 2017.

135 Du serment. Différence entre le serment politique et le serment judiciaire, op.cit.

136 Selon l'expression de la Cour européenne des droits de l'homme dans ses arrêts Piersack et de Cubber c/ Belgique des $1^{\mathrm{er}}$ octobre 1982 et 26 octobre 1984, l'impartialité des juges est la condition même de «la confiance que les tribunaux se doivent d'inspirer aux justiciables dans une société démocratique ».

137 De GOUTTES (R.), «L'impartialité du juge. Connaître, traiter et juger : quelle compatibilité? », RSC, 2003 p. 63. 
subit pas de pressions. Est impartial, celui qui n'a pas de préjugé. Comme l'observe Guinchard, "pour les juges, l'exigence d'indépendance se traduit par l'élaboration d'un statut protecteur, dont l'inamovibilité est l'un des éléments essentiels, et par l'interdiction des immixtions dans l'exercice de leurs fonctions ».

\section{La dérision du contrôle de la morale par le juge constitutionnel}

Selon le doyen Duguit, le juge « constate le droit», mais il «ne fait pas acte de volonté à proprement parler » puisqu'il «ne peut pas mettre sa volonté à la place de l'opération logique, qui est dominée par une mineure et une majeure, suivant les lois de la pensée humaine ». ${ }^{138}$ Aujourd'hui, nul ne doute plus du pouvoir normatif du juge. ${ }^{139}$ Alors que tout le débat sur la production normative d'origine jurisprudentielle semble être mûri, ${ }^{140}$ la question est à nouveau réactivée dans une perspective de son appréciation à l'aune de la règle morale. Le juge recourt à la règle morale non seulement pour approfondir les textes, mais parfois pour dire entièrement le droit. ${ }^{141} \mathrm{Il}$ est souverain pour déterminer le sens des textes et inventer des normes (A). De plus, il contribue énormément à la moralisation de la vie politique (B).

\section{A. L'office du juge mué en un pouvoir normatif concurrent}

L'exclusivité de la production du droit par l'Etat découvre une portée naturelle dans le critère formel des normes : le caractère juridique d'une norme se déduit de son origine institutionnelle. A la faveur du positivisme, les règles juridiques peuvent être ordonnées selon la hiérarchie des sources du droit. Aujourd'hui, la jurisprudence trouve progressivement sa place dans cette hiérarchie. ${ }^{142}$ L'analyse de la doctrine révèle que les principes issus des arrêts des juridictions suprêmes se glissent dans la structure pyramidale du droit. Mais, face

138 DUGUIT (L.), «L'acte administratif et l'acte juridictionnel », RDP, 1906, p. 450-451.

139 Aussi, exerce-t-il son activité dans un cadre saturé d'obligations. Les théoriciens formalisent ces contraintes dans un discours savant qui définit, autorise et limite les prérogatives judiciaires.

140 "Que le Conseil constitutionnel exerce un certain pouvoir normatif, comme toute juridiction suprême est naturellement appelée à le faire, voilà qui n'est guère contestable. Sans doute n'est-il pas à cet égard dans la même situation que le Conseil d'État, qui a élaboré par voie jurisprudentielle des pans entiers d'un droit administratif dont les lois et règlements ne fournissaient pas la matière : dans sa mission de contrôle de la constitutionnalité des lois, le juge constitutionnel s'appuie au contraire sur des normes de référence écrites et circonscrites à ce que contient la Constitution, y compris son Préambule ». Voir ARRIGHI de CASANOVA (J), " Pouvoir normatif du Conseil constitutionnel et stabilité de la norme », Cahiers du Conseil constitutionnel n 24 (Dossier : Le pouvoir normatif du juge constitutionnel) - juillet 2008.

141 Ainsi, dispose-t-il alors «d'un pouvoir considérable, puisque c'est lui qui produit la norme qu'il est censé appliquer ». Rien ne semble faire obstacle au pouvoir du juge.

142 FORRAY (V.), « La jurisprudence, entre crise des sources du droit et crise du savoir des juristes ", RTD Civ., 2009, p. 463. 
au recours des juges à la règle morale, il importe de revisiter la théorie kelsénienne puis d'examiner la régression de la loi dans l'ordre normatif.

\section{La résurgence du débat sur le rejet traditionnel du pouvoir normatif du juge}

Le juge est titulaire d'un pouvoir normatif. L'affirmation à l'heure actuelle évidente, incontestable. L'idée selon laquelle le juge ne fait que dire le droit mais ne le crée jamais est sans aucun doute dépassée. Il s'agit là d'une approche fictive, à la limite puérile; la théorie de Montesquieu selon laquelle le juge « n'est pas plus que la bouche par laquelle le droit s'exprime » est pareillement discréditée. ${ }^{143}$ Toutefois, l'irruption de la morale, valeur non neutre, dans l'office du juge, peut amener à s'interroger voire revisiter les thèses qui s'opposent à l'admission du pouvoir normatif du juge.

Il faut rappeler à l'instar de Atias que « le positivisme juridique se défend de toute impureté idéologique et métaphysique (...) : il parlerait seul du vrai droit et toute autre théorie serait condamnée à errer dans le rêve, dans les choix idéologiques et métaphysiques $»^{144}$ et qu'en réalité, selon le "dogme général du positivisme », le juriste ne doit jamais "discerner le juste », il doit demeurer, précise Villey, " neutre; peu lui importe que [les] textes [qu'il étudie] visent la domination de la race germanique sur le monde, l'élimination des bourgeois, l'épanouissement des libertés, ailleurs la justice ». ${ }^{145}$

Tout comme les juridictions ordinaires sont « la bouche de la loi », le Conseil constitutionnel est celle de la Constitution; et, tout comme leur jurisprudence, la sienne va au-delà de la stricte application d'un texte qui ne peut tout dire ni tout prévoir. Dans cette approche, une incursion sur la « lecture morale du juge ${ }^{146}$ est nécessaire. On doit ainsi tenir pour acquis que si, selon une formule chère au doyen Vedel, la loi n'exprime la volonté générale que dans le respect de la Constitution, cela signifie que le législateur se trouve astreint au respect d'une jurisprudence constitutionnelle qui explicite, prolonge et complète la loi fondamentale. Mais en recourant à la règle morale, ne donne-t-il pas l'opportunité à de nouvelles critiques de la part de tous ceux qui contestent le pouvoir normatif du juge? De plus,

143 BARAK (A)., « L'exercice de la fonction juridictionnelle vu par un juge : le rôle de la Cour suprême dans une démocratie », Revue française de droit constitutionnel 2006/2 ( $\left.n^{\circ} 66\right)$, p. 229.

144 ATIAS (C.), « Fonder le droit ».

145 VILley (M.), Philosophie du droit. Définitions et fins du droit t.1. Les moyens du droit t. 2, Dalloz, Réédition, 2001, p. 137.

146 Le contrôle de constitutionnalité des lois en matière de droits et libertés ne pourrait être considéré comme légitime qu'à la condition que les juges procédant à ce contrôle puissent être parfaitement neutres (c'est-à-dire dans la mesure où a) leurs opinions morales personnelles n'infléchissent pas le droit et où b) ils ne sont que « la bouche de la loi ». Par une telle neutralité, les juges respecteraient ainsi l'autorité législative, seule détentrice de la légitimité démocratique; Or, une telle neutralité ne s'avère possible que si la formulation des droits et libertés constitutionnalisés est à ce point précise et détaillée qu'il n'y a plus de place pour leur interprétation; Mais comme une telle précision n'est pas envisageable, les juges ne peuvent faire preuve de neutralité. BEGIN (L)., «L'impartialité des juges et la lecture morale des droits », Les Cahiers de droit, 38(2), 1997, p. 418. 
le recours à la règle morale par le juge est l'occasion de discuter à nouveau de la « querelle » entre les tenants du positivisme et ceux du droit naturel. Pour Troper, dans le langage courant, on désigne comme juge " positiviste » celui qui se limite à invoquer les textes adoptés par une autorité compétente, sans en écarter aucun et sans même fonder le choix entre plusieurs textes sur des raisons tirées de la morale, et comme jusnaturaliste celui qui au contraire écarte un texte ou choisit entre plusieurs pour ces raisons morales. ${ }^{147}$ Troper évoque dans « La pyramide est toujours debout! Réponse à Paul Amselek », une préoccupation fondée d'une part, sur la nécessité de distinguer la norme produite par le juge constitutionnel de celle générée par la catégorie des juges ordinaires, et d'autre part, celle d'apprécier la norme d'origine jurisprudentielle fondée sur des interprétations morales. ${ }^{148}$

Par ailleurs, le recours à la règle morale va nourrir également les tensions inhérentes à la séparation des pouvoirs, notamment la relégation du pouvoir judiciaire derrière le législatif et l'exécutif. Or, notre époque s'inscrit dans une perspective inversée : les juges contrôlent désormais de manière approfondie le respect par la loi des normes constitutionnelles et internationales ainsi que tous les actes et toutes les activités des décideurs publics.

Même si la «pyramide est toujours debout ", pour reprendre les propos de Troper, l'intrusion ou peut-être l'invitation de la règle morale dans l'office du juge semble porter un coup sérieux à la théorie pure du droit ou " prétendument pure » de Kelsen. ${ }^{149} \mathrm{Il}$ considère qu'il n'est pas d'autre droit que le droit positif et assigne au juriste le devoir de se tenir à l'écart de son objet en se contentant de le décrire de lege lata. ${ }^{150}$

La question de la normativité est une question récurrente en droit public. Nul n'ignore les querelles doctrinales sur la valeur juridique des principes généraux du droit. ${ }^{151}$ En effet

147 TROPER (M.), « Le réalisme et le juge constitutionnel », Cahier du Conseil constitutionnel n 22 (Dossier : Le réalisme en droit constitutionnel) - juin 2007. Voir aussi, AMSELEK (P.), « Réflexions critiques autour de la conception kelsénienne de l'ordre juridique », RDP, 1978, p. 5-19.

148 RDP, 1978, p. 1523-1536.

149 Selon KELSEN, les règles sont structurées dans un ordre hiérarchique. Il va établir une sorte de pyramide comportant différents niveaux, chaque norme occupant un niveau différent et dans laquelle les normes se trouvant au niveau le plus bas tirent leur validité des normes se trouvant au niveau le plus haut. Au sommet de la pyramide il y a une règle spéciale, la Grundnorm en allemand, une règle fondatrice, qui est la norme fondamentale donnant sa validité à la totalité du système.

150 Il adopte ainsi, dans sa plus parfaite expression, une posture que d'autres juristes avant lui avaient observée de façon moins radicale, à l'instar de CARRE de MALBERG, de JELLINEK, de LABAND ou de BERGBOHM. Confère. VIALA (A.), « Le positivisme juridique : Kelsen et l'héritage kantien », Revue interdisciplinaire d'études juridiques 2011/2 (Volume 67), p. 95.

151 Nul n'ignore non plus le débat sur leur valeur juridique des préambules des Constitutions. Tetang F. affirme en ce qui concerne la valeur juridique des préambules, que certaines Constitutions en sont dotées mais, paradoxalement, restent muettes sur la valeur juridique. Aussi, va-t-il ajouter qu'une partie de la doctrine n'y a vu qu'un ensemble de déclarations de principes dépourvues de force juridique et par conséquent, dénuées de toute normativité et même de force normative. La morale ou la règle morale est sans doute une norme, mais une norme dépourvue de sanction. Il y 
sont appelés principes généraux du droit des principes formulés par le juge administratif, soit à partir de textes, soit même sans texte et dont il impose le respect à l'administration.

Le recours du juge à la règle morale incite à une rupture dans les modes d'acquisition et de progression du savoir juridique. La réponse à une question en droit ne peut plus se satisfaire de la connaissance synthétique du droit positif; le droit ne peut plus être maitrisé par la connaissance exhaustive de ses sources. ${ }^{152}$

Découvrant un litige, des actes, un dossier, entendant des plaidoiries, le juge a nécessairement une première intuition qui peut influencer sa décision. Il s'agit de ses convictions personnelles, de ses opinions, de son tempérament ${ }^{153} \ldots$ L'influence de la règle morale sur toutes décisions du juge est difficile à éviter. Et comme le précise Chazal : «On ne peut pas dire que la matière juridique se caractérise par une certitude absolue, mais on ne peut pas dire non plus qu'elle n'en contienne aucune... On ne peut pas croire que la bonne décision est toujours découverte, mais on ne peut pas affirmer qu'elle ne l'est jamais $\gg{ }^{154} \mathrm{Il}$ va de soi que lorsque le juge se mue en un pouvoir législatif concurrent, cela peut parfois entrấner une insécurité juridique.

\section{La crainte de l'insécurité juridique}

« Si la "jurisprudence" peut assurément changer, il faudra bien un jour se demander comment concilier ses variations avec les exigences de la justice " ${ }^{155}$ à savoir la sécurité juridique. L'insécurité juridique peut être aussi générée par l'invitation de la règle morale dans l'office du juge. Le concept même de sécurité juridique est un concept introuvable, sinon insaisissable. Certains auteurs, comme Mathieu, vont même jusqu'à qualifier le concept de « clandestin ». ${ }^{156}$

a lieu de se demander si l'appropriation de la morale par le juge ne transforme pas cette dernière en une règle positive. TETANG $(\mathrm{F}$.), « La normativité des préambules des constitutions des États africains d'expression française », Revue française de droit constitutionnel 2015/4 ( $\left.{ }^{\circ} 104\right), \mathrm{p}$. 953.

152 Ce qui illustre bien l'idée selon laquelle le droit ne peut se réduire à un « ensemble de règles » puisque le juriste est incapable d'établir avec certitude ce qui est du droit et ce qui ne l'est pas.

153 ATIAS (C.), Philosophie du droit, PUF, 20196, p. 345 et 346.

154 CHAZAL (J.-P.), « Philosophie du droit et théorie du droit, ou l'illusion philosophique », in $\mathrm{Ar}$ chives de philosophie du droit, 45, 2001, p. 331.

155 ATIAS (C.), note sous Cass. civ. 1ère, 21 mars 2000, D. 2000, p. 593.

156 Il comporte pourtant de nombreuses applications essentielles dans notre droit : prévisibilité de la loi, clarté et accessibilité de la norme, stabilité des situations juridiques, intelligibilité des lois, exigences encore tout récemment rappelées par des décisions du Conseil constitutionnel comme du Conseil d'État. MATHIEU (B.), « La sécurité juridique, un principe constitutionnel clandestin mais efficient », Mélanges Patrice GELARD, Montchrestien, 2000, p. 301. Voir aussi MALAURIE (P.), «L'intelligibilité des lois », Pouvoirs 2005/3 (n 114), p. 131. 
La notion de sécurité juridique est absente, en tant que telle, de notre corpus constitutionnel. Elle ne figure ni dans le texte de la Constitution de 1958, ni dans celui du Préambule de 1946, ni même dans la Déclaration des droits de l'homme et du citoyen de 1789.

Pour le Conseil constitutionnel, la sécurité juridique se rattache cependant à deux notions qui, bien que proches de celle de sécurité juridique, ne coïncident pas exactement avec celle-ci. La première est la notion de " sûreté » qui, est l'un des droits naturels et imprescriptibles de l'homme avec la liberté, la propriété et la résistance à l'oppression. ${ }^{157}$ Mais, dans la Déclaration de 1789, la notion de sûreté correspond principalement à la notion $d$ 'habeas corpus, comme le précise expressément l'article 7 : «Nul homme ne peut être accusé, arrêté ni détenu que dans les cas déterminés par la loi, et selon les formes qu'elle a prescrites ». La seconde notion est la notion de «garantie des droits » : «Toute société dans laquelle la garantie des droits n'est pas assurée, ni la séparation des pouvoirs déterminée, n'a point de constitution $\gg .{ }^{158}$

Au cours du séminaire franco-brésilien organisé par la Cour de Cassation en France le 19 septembre 2005 sur la sécurité juridique, Dutheillet de Lamothe en présentant « Regards croisés sur la sécurité juridique » affirme que «face au désordre du droit, le principe de sécurité juridique apparaît comme la dernière branche à laquelle s'accrochent les juridictions suprêmes pour maintenir un semblant d'ordre et permettre au droit de remplir la mission qui est normalement la sienne ».

Ces inquiétudes ont été révélées, dès 1991, par le Conseil d’État, dans ses « considérations générales » consacrées à la sécurité juridique sur cette complexité du droit, caractérisée par la prolifération désordonnée des textes, l'instabilité croissante des règles et la dégradation manifeste de la norme, n'ont eu que peu d'effets, à l'exception de progrès significatifs en matière d'accessibilité des textes, principalement par la codification et par la création de bases de données publiques.

De même, Malaurie, dans «L'intelligibilité des lois " ${ }^{159}$ affirme que "l'obscurité des lois rend le droit imprévisible, en fait un instrument de l'arbitraire, indulgent envers les habiles et les puissants, impitoyable envers les faibles et les maladroits, une source permanente de conflits, de verbalismes, de procédures judiciaires interminables; elle est un des moyens de mettre fin à l'état de droit, le plus pitoyable parce que c'est l'inintelligence qui le fait disparaître : une loi inintelligible est une mascarade juridique $\gg{ }^{160}$ La pénétration de la règle morale dans l'office du juge va métamorphoser les règles de la construction jurisprudentielle. Coincé entre l'obligation d'éviter le déni de justice ${ }^{161}$ et l'exigence de ne pas

157 Voir l'article 2 de la Déclaration de 1789.

158 Voir l'article 16 de la Déclaration.

159 Pouvoirs 2005/3 (n 114), p. 131.

160 Seule la loi claire, simple, limpide, transparente, compréhensible de tous peut être respectée, devenir efficace et assurer ce que l'on peut attendre du droit : la justice, l'ordre, la prévisibilité, la sécurité, le bien-être, la paix et peut-être le bonheur.

161 «Le juge qui refusera de juger, sous prétexte du silence, de l'obscurité ou de l'insuffisance de la loi, pourra être poursuivi comme coupable de déni de justice ». Art. 4 du code civil. 
se muer en un pouvoir normatif, ${ }^{162}$ le juge va finir par s'émanciper et s'affranchir de cette délimitation quelque peu irréaliste, ceci au détriment de la loi.

Mais la force symbolique de la loi ne favorise pas la recherche de solutions alternatives à l'élaboration de normes, et conduit à faire passer toute réforme importante par cet instrument. L'une des caractéristiques de l'exception française, liée aux colbertismes de droite ou de gauche, réside en effet dans la propension à attendre des miracles de la loi, à la juger en fonction de ses motifs plutôt que de ses conséquences, et à faire appel à l'État législateur à tout propos, en escomptant de la loi des résultats à la fois prompts, bienfaisants et exempts d'effets pervers. ${ }^{163}$

Le Conseil d'État va jusqu'à discerner dans la complexité croissante des normes une menace pour l'État de droit. ${ }^{164} \mathrm{Vu}$ du continent africain, la question mérite d'être approfondie. Le processus d'édification de l'Etat de droit risque de s'enliser, si l'émancipation du juge par rapport à la loi n'est pas encadrée. Déjà contesté, ${ }^{165}$ le juge africain serait davantage exposé si son office est fondé sur des règles subjectives qu'il est seul à pouvoir interpréter.

Le juge s'est émancipé et sans doute un peu trop aux yeux de certains auteurs. On connait bien la théorie du gouvernement des juges et même celle de la « saison des juges » chère à Heuze. ${ }^{166}$ Ce qui est certain, c'est que le temps où la jurisprudence était qualifiée d'illégitime est révolue. Le propos de Raynaud selon lequel « dès lors que la souveraineté a été restituée à la nation, la jurisprudence $n^{\prime}$ a plus aucune raison d'être ${ }^{167}$ est d'une autre époque. La question de la règle morale revient encore sous une autre forme, celle de la moralité. Le dessein de l'édification d'un système démocratique assorti de celui de l'Etat de droit ${ }^{168}$ serait dans ces conditions compromis. Ce faisant, on pourrait se demander si le pas vers le gouvernement des juges n'est pas en train d'être esquissé. ${ }^{169}$

162 « Il est défendu aux juges de prononcer par voie de disposition générale et réglementaire sur les causes qui leur sont soumises ». Art. 5 du code civil.

163 Rapport du Conseil d'Etat, « Sécurité juridique et complexité du droit », 2006, p. 231.

164 Conseil d'État, Rapport public 2006 : Sécurité juridique et complexité du droit, Paris, La Documentation française, p. 233 et s.

165 «Si le juge est un organe de l'État assez connu et la justice une institution bien installée, les rapports entre ceux-ci et les particuliers, susceptibles de les solliciter, cachent des réalités que l'on ne soupçonne généralement pas, et que l'on ne peut découvrir qu'aux termes d'une observation prudente et attentive des conditions d'exercice et de fonctionnement de la justice en Afrique. C'est dans cette perspective qu'il faut envisager l'isolement du juge ». BADARA FALL (A).

166 HEUZE (V.), « À propos du rapport sur les revirements de jurisprudence, une réaction entre indignation et incrédulité », JCPG, 2005, I, 130.

167 RAYNAUD (P)., La loi et la jurisprudence des lumières à la révolution française, Arch. Phil. dr., t. 30 , p. 62.

168 Voir les préambules des Constitutions des Etats de l'Afrique adoptées après les années 1990.

169 HOUNAKE (K.), Les juridictions constitutionnelles dans les démocraties émergentes de l'Afrique noire francophone: Les cas du Bénin, du Gabon, du Niger, du Sénégal et du Togo, Thèse, Université de Lomé, 2012, p. 126. Voir également FALL (A. B.), « Le juge constitution- 


\section{B. L'office du juge à l'aune de la gouvernance politique}

La liberté dont dispose le juge accroit la polémique relative au recours à la morale dans sa démarche. Si une certaine forme de neutralité ne peut être respectée par le juge, c'est la certitude même que la démocratie constitutionnelle, apparue après 1990, risque d'être compromise. ${ }^{170}$

Aussi, le juge sera-t-il accusé de s'immiscer dans l'action de l'exécutif et du législatif ou de vouloir s'ériger en moralisateur de la vie publique. ${ }^{171}$ Mais en France, le Conseil constitutionnel dispose potentiellement à l'égard des lois d'une puissance plus forte que la Cour européenne des droits de l'homme. ${ }^{172}$ Ce qui fait dire à Blacher que le Conseil constitutionnel est sans doute une juridiction mais surtout un législateur négatif. De Bechillon réactualise le concept du gouvernement des juges ${ }^{173}$ et rappelle que l'appellation " gouvernement des juges $»^{174}$ est déposée depuis trop longtemps dans l'imaginaire général pour que l'on ait une bonne chance de déboucher sur quoi que ce soit d'utile avec elle. ${ }^{175}$ Ainsi, des législations moralement condamnables et qui soulèvent d'évidents problèmes de conscience, ont toujours existé et ont traversé l'histoire des nations. ${ }^{176}$

nel, artisan de la démocratie en Afrique? ", Communication au Colloque de l'Association Française de Droit Constitutionnel (juin 2005, Montpellier).

170 BEGIN L., «L'impartialité des juges et la lecture morale des droits », Les Cahiers de droit, 38(2), 1997, p. 419.

171 En France, depuis le $1^{\text {er }}$ mars 2010, le Conseil constitutionnel peut contrôler et, surtout, abroger une loi. Il s'agit selon BLACHER (P.) d'une véritable révolution juridique. Officiellement, le Conseil constitutionnel est en mesure d'abroger la loi. Certes, ce pouvoir de législation négatif est le résultat d'un dialogue des juges dans la mesure où c'est à la suite d'une demande, filtrée par les plus hautes juridictions, que l'abrogation intervient. BLACHER (P.), « Vers un gouvernement du juge constitutionnel? », AJDA 2010, p. 465.

172 BLACHER (P.), « Vers un gouvernement du juge constitutionnel? », AJDA 2010, p. 465

173 LAMBERT (E.), Le gouvernement des juges et la lutte contre la législation sociale aux EtatsUnis. L'expérience américaine du contrôle judiciaire de la constitutionnalité des lois, Paris, Girard, 1921, Dalloz, 2005, $276 \mathrm{p}$.

174 Selon TROPER, l'expression gouvernement des juges, a été introduite en France par Edouard LAMBERT, qui la fait figurer dans le titre même de son ouvrage. Il qualifie ainsi le rôle de la Cour Suprême des Etats-Unis. La formule est employée aujourd'hui, le plus souvent avec une connotation péjorative, à chaque fois qu'on veut critiquer le pouvoir jugé excessif des juges, plus spécialement, mais pas exclusivement, celui des juridictions constitutionnelles. « Le bon usage des spectres. Du gouvernement des juges au gouvernement par les juges », Mélanges en l'honneur de Gérard CONAC, Economica, p. 49.

175 Recueil Dalloz 2002 p. 973.

176 Cette préoccupation s'attèle bien avec la question de la morale dans la vie politique, qui d'ailleurs, est aussi vieille que les sociétés politiques de la Grèce antique. 
1. La moralisation de la vie publique au cœur de l'office du juge

Selon Dworkin, la lecture morale des droits et de la Constitution est fréquente dans les juridictions, même si les juges refusent de le reconnaître. Il y aurait en fait un écart considérable entre la pratique constitutionnelle et la théorie constitutionnelle qui rejette fortement cette lecture. C'est que la lecture morale semble reconnaître aux juges un trop grand pouvoir d'imposer leurs propres convictions morales à la collectivité. ${ }^{177}$

La moralisation de la vie publique passe aujourd'hui par une forte judiciarisation $\mathrm{du}$ secteur politique et du monde des affaires. La montée en puissance du Conseil constitutionnel a judiciarisé le processus législatif. De plus, la multiplication des affaires de corruption qui perturbent et mettent un terme à des carrières politiques, " remplissent » aujourd'hui l'office du juge. ${ }^{178}$ Certaines professions qui jouissaient d'une immunité de fait se sont vues dans les mêmes années attraites devant la justice : les médecins poursuivis par leurs patients, les autorités militaires attaquées en justice par des familles de soldats morts au combat, ou encore les congrégations religieuses à qui des prêtres défroqués demandent la requalification de leurs vœux en contrats de travail. ${ }^{179}$ Cette judiciarisation frappe d'autant plus les esprits qu'elle concerne des institutions symboliques ${ }^{180}$ qui voient leur statut symbolique diminué.

De nombreux auteurs, ont souligné à quel point la vie publique est, désormais, « saisie par le droit $» .{ }^{181}$ La juridicisation de la vie publique dans les démocraties est flagrante, dans la mesure où, aujourd'hui, les relations entre juges et acteurs de la vie publique font l'objet d'un vif débat dans ces Etats. ${ }^{182}$ La justice constitutionnelle y apparaît comme un instrument de modération de l'exercice du pouvoir politique, et la multiplication des " affaires » $\mathrm{y}$ a mis à nu la «pénalisation » de la vie politique. De plus, l'on assiste à une constitutionnalisation de la vie politique ${ }^{183}$. Ainsi, en 1978, les constituants espagnols ont veillé à ce que le travail législatif nécessaire pour réussir la transition démocratique ne soit pas réduit à néant

177 BEGIN (L.), op. cit., p. 421.

178 Voir La prudence et l'autorité, l'office du juge au XXème siècle, Rapport de la mission de réflexion confiée par Madame Christiane TAUBIRA, garde des Sceaux, à l'Institut des hautes études sur la justice, sur l'évolution de l'office du juge et son périmètre d'intervention. RAPPORT DE L'IHEJ, MAI 2013.

179 Idem.

180 La souveraineté des États, le Parlement, la médecine, l'armée, la religion...

181 MONTES (J.), «Le retour du « gouvernement des juges ». Analyse comparée de la juridicisation de la vie politique dans la France et l'Espagne contemporaines », RSC, 2002 p. 293.

182 Idem.

183 Parce que la Constitution est la norme suprême, le contrôle de son respect ne saurait être abandonné au juge ordinaire comme c'est le cas dans le modèle diffus apparu aux Etats-Unis en 1803 avec l'arrêt Madison vs Marbury. Or, le choix du modèle concentré de contrôle de constitutionnalité obéit, aussi, à des motifs politiques.; Voir aussi, FRANÇOIS (B.), Le régime politique de la Ve République, Paris, La découverte, col. Repères, 1999, p. 109. 
par un appareil judiciaire longtemps assujetti au régime franquiste. ${ }^{184}$ Vingt ans plus tôt, la France du général de Gaulle optait pour le modèle concentré dans un souci de mieux contenir le pouvoir législatif. Ce modèle a été repris par la majorité des Etats africains d'expression française. Le Togo, le Bénin, le Gabon, la Côte d'Ivoire, le Sénégal, ont adopté un modèle similaire à celui de la France.

Un modèle critiqué parfois par la doctrine qui relève ses ambiguïtés. Diakhate souligne la vulnérabilité des garanties statutaires en revenant sur les modes de désignation des juges constitutionnels. Il note également la dépréciation de l'autorité des décisions, une dépréciation marquée par la faiblesse des solutions jurisprudentielles et la précarisation du pouvoir juridictionnel. ${ }^{185}$ Dans cette dynamique, l'Afrique sans verser dans le mimétisme va s'engager dans la recherche de voies adaptées pour asseoir une construction constitutionnelle originale. ${ }^{186}$ En effet, de nombreux organes chargés d'exercer le rôle de contre-pouvoirs ont été mis en place afin de garantir dans les nouveaux systèmes une stabilité juridico-politique. ${ }^{187}$ C'est précisément dans ce contexte de regain constitutionnel que le constituant, dans certains Etats d'Afrique, va procéder à la création de nouvelles juridictions constitutionnelles autonomes et spécialisées, à la place des anciennes Chambres et Sections constitutionnelles ${ }^{188}$, mettant ainsi, selon le professeur Conac, « un point d'honneur à organiser ou à réorganiser le contrôle de constitutionnalité ». ${ }^{189}$

La moralisation de la vie publique suppose qu'un certain nombre de valeurs morales soient respectées. ${ }^{190}$ L'arsenal juridique et institutionnel en place devrait garantir une activité politique exempte de vice moral. Le principe de séparation des pouvoirs, le respect des droits de l'homme et des libertés fondamentales, l'existence d'une organisation judiciaire relativement bien structurée, ${ }^{191}$ la consécration d'une juridiction constitutionnelle et d'une Haute Cour de Justice ${ }^{192}$ constituent autant d'instruments qui devraient contribuer à garantir

$184 \operatorname{MONTES}$ (J.), « Le retour du « gouvernement des juges ». Analyse comparée de la juridicisation de la vie politique dans la France et l'Espagne contemporaines », op. cit.

185 DIAKHATE (M.), « Les ambigüités de la juridiction constitutionnelle dans les Etats de l'Afrique noire francophone », RDP, 2015, p. 785.

186 DIALLO (I.), « A la recherche d'un modèle africain de justice constitutionnelle » Essais in Annuaire International de Justice Constitutionnelle, XX, 2004 p. 93-120.

187 DIALLO (I.), " A la recherche d'un modèle africain de justice constitutionnelle », Essais in $A n$ nuaire International de Justice Constitutionnelle, XX, 2004, p. 94.

188 SY (P.) (M.), Le développement de la justice constitutionnelle en Afrique noire francophone : les exemples du Bénin, du Gabon et du Sénégal, Thèse, Dakar,1998, p. 27.

189 CONAC $(\mathrm{G})$. « Etat de droit et démocratie » in L'Afrique en transition vers le pluralisme politique, p. 497.

190 L'égalité, la légitimité, l'honnêteté du personnel politique, la transparence et la réalisation de l'intérêt général sont les éléments décisifs d'une vie politique saine.

191 Voir l'ordonnance $\mathrm{n}^{\circ} 78-35$ du 7 septembre 1978 portant organisation judiciaire au Togo. Selon l'article $1^{\mathrm{er}}$, au Togo la justice est rendue par la Cour Suprême, des Cours d'Appels, des tribunaux de première instance.

192 Voir article 126 de la Constitution de la République du Togo. 
la moralisation de l'exercice de l'activité politique. L'actualité politique récente démontre que les mécanismes juridiques et institutionnels existants sont loin d'assainir le milieu politique. ${ }^{193}$ Ainsi, le juge se trouve interpelé et sa volonté de dire le droit peut prendre une tournure discutable.

2. La controverse sur les prétendues usurpations des pouvoirs par le juge

Une précision s'impose à ce niveau de la réflexion, c'est de savoir, d'une part, le moment exact où les juges usurpent les prérogatives d'une autre institution et d'autre part, la signification d'un concept « scientifique » du gouvernement des juges. Cela veut dire qu'il faut disposer de critères permettant de dire quand les juges font quelque chose qu'ils ne devraient pas faire. Ceci permettra d'identifier le moment précis où les juges gouvernent vraiment. ${ }^{194}$ Le doyen Vedel, à propos du Conseil constitutionnel français, précisait que « $L a$ plus sûre garantie de la stabilité, sinon de la pérennité de la jurisprudence constitutionnelle, se trouve dans le fait que le Conseil constitutionnel, largement mis en garde contre le danger du 'gouvernement des juges' ne s'estime pas maître des sources du droit constitutionnel. On ne peut guère citer de motif d'une de ses décisions qui ne se référerait pas avec 'précision à un texte de valeur constitutionnelle'... ». De même, plusieurs auteurs, tels Rawls ou Eisenmann, ont pointé du doigt le risque d'une dérive politique de la justice constitutionnelle ${ }^{195}$.

Dans ses contributions sur le pouvoir dans l'Etat, Beaud explique que le principe du constitutionnalisme désigne la limitation du pouvoir par le droit, la subordination de l'activité politique à la constitution, ${ }^{196}$ une subordination assurée par la justice constitutionnelle. Les règles de droit sont ainsi, pour reprendre les analyses de Lagroye, constitutives d'une « forme de domination légal-rationnelle », et les rejeter serait se placer d'une certaine ma-

193 La destitution de l'ancienne Présidente de la République fédérale du Brésil et les déboires actuels du président en fonction, l'arrestation du Maire de Dakar au Sénégal et les scandales des emplois fictifs en France illustrent bien la situation. Voir aussi, Le Monde du 31 août 2016, « Brésil : la présidente Dilma Rousseff destituée »,

http://www.lemonde.fr/ameriques/article/2016/08/31/bresil-la-presidente-dilma-rousseff-destitue e_4990645_3222.html consulté le 27 juin 2017.

http://www.lemonde.fr/afrique/article/2017/03/08/accuse-de-detournement-de-fonds-le-maire-dedakar-a-ete-inculpe-et-ecroue_5090873_3212.html consulté le 27 juin 2017.

http://www.bfmtv.com/politique/affaire-penelope-fillon-un-scandale-plusieurs-issues-possibles-1 090550.html consulté le 27 juin 2017.

194 De BECHILLON (D.), « Le gouvernement des juges : une question à dissoudre », Recueil Dalloz, 2002 p. 973.

195 RAWLS (J.), Théorie de la justice, Paris, Seuil, col. Points essai, 1999, p. 257. EISENMANN (C.), La justice constitutionnelle et la Haute Cour constitutionnelle d'Autriche, Paris, Economica, col. Droit public positif, 1986, p. 105.

196 BEAUD (O.), La puissance de l'Etat, Paris, PUF, col. Léviathan, 1994, p. 19. 
nière hors de la collectivité. ${ }^{197}$ Bien qu'impartiales et indépendantes, les juridictions constitutionnelles se sont vues confier une mission éminemment politique : garantir le respect de la norme suprême. ${ }^{198} \mathrm{Un}$ exercice qui peut les amener à recourir à la règle morale ou, dans une autre hypothèse, se transformer en une cour qui rend des "jurisprudence excessives $\gg .{ }^{199}$

Pour de nombreux citoyens, le juge qui gouverne est un usurpateur. Cet excès lui permet de confisquer à son profit une compétence fondamentalement politique, qui ne peut ni ne doit lui appartenir. ${ }^{200}$ Dans ce contexte, la morale s'invite dans l'office du juge. Ce n'est plus le juge qui se réfère aux règles morales dans sa démarche, c'est sa propre moralité qui fait l'objet de critique. Un juge dont l'activité remet permanemment en cause les initiatives de l'exécutif est considéré comme un activiste, auteur de coup d'Etat permanent, pour emprunter les propos du président Mitterrand. ${ }^{201}$ Analysée sous cet angle, la question n'est pas nouvelle. Ce qui est nouveau, c'est justement la moralité du juge qui peut déterminer le choix de cette émancipation, ou, pire, de cet activisme. L'idéal, c'est que le juge soit impartial. Dans ses réflexions sur les normes garantes d'un procès de qualité en Europe, Guinchard souligne la nécessité de l'impartialité du juge. ${ }^{202}$ La présence de la morale dans l'office et le souci de bonne moralité dont le juge doit faire preuve, vont irriguer la controverse sur la prétendue transformation de la fonction prétorienne. L'imprécision de la règle morale et la subjectivité du concept de moralité accroissent la liberté d'interprétation du juge et ce dernier peut inconsciemment ou non tomber dans les travers de l'excès. Troper prévenait déjà que la liberté juridique du juge est totale, puisqu'il peut créer et recréer à chaque instant des normes et des relations entre normes, c'est-à-dire qu'il est le maître du système ju-

197 LAGROYE (J.), Sociologie politique, Paris, Presses de Sciences Po, Dalloz, col. Amphitor, 3e éd. 1977 , p. 142.

198 GREWE (C.), RUIZ FABRI (H.), Droits constitutionnels européens, Paris, La documentation française, 1995 , p. 75.

199 Evoquer une jurisprudence excessive implique de déterminer, pour chaque affaire, ce qu'il aurait été " raisonnable » de juger. Or cette notion de " raisonnable » peut fortement varier d'une personne à l'autre. HOURQUEBIE ((F.) et PONTHOREAU (M.-C.) dir., La motivation des décisions des cours suprêmes et cours constitutionnelles, Bruylant, 2012, p. 80.

200 De BÉCHILLON, op. cit.

201 Il ne voit dans le Conseil constitutionnel qu'un « garçon de course [...], un agent empressé du pouvoir exécutif. Voir, Le Coup d'Etat permanent, Plon, Paris, 1964, p. 140-141. Voir aussi MENDES FRANCE (P.), La République Moderne, Coll. Idées, Gallimard, Paris, 1966, p. 44, cité par HOUNAKE (K.), Les juridictions constitutionnelles dans les démocraties émergentes de l'Afrique noire francophone: Les cas du Bénin, du Gabon, du Niger, du Sénégal et du Togo, Thèse, Université de Lomé, 2012, p. 11.

202 GUINCHARD (S.), «Les normes européennes garantes d'un procès de qualité », in Marie-Luce CAVROIS, Hubert DALLE et Jean-Paul JEAN (dir.), La qualité de la justice, Paris : La Documentation française, 2002, p. 64. 
ridique. ${ }^{203}$ Mais le juge peut ainsi devenir inflexible à l'endroit des autres institutions. ${ }^{204}$ C'est pourquoi l'auteur précise que si cette conclusion paraît fortement exagérée, il faut retenir surtout qu'il n'y a pas dans la réalité un seul interprète, mais une pluralité, et qu'ils forment un système. Et ce système est constitutif de contraintes qui empêchent chacun d'exercer complètement et à chaque instant son pouvoir discrétionnaire. Le plus surprenant est que ce pouvoir d'abrogation ait été accordé à un juge - fût-il constitutionnel - à l'occasion d'une révision dite de « modernisation des institutions » censée restaurer le Parlement en France. Les parlementaires, réunis en congrès, ont ainsi adopté un dispositif brisant le principe républicain d'immunité de la loi promulguée.

Ce pouvoir ainsi accordé au Conseil constitutionnel en France, ne suscite guère d'émotion au sein de la communauté des « constitutionnalistes ». L'entrée de la norme constitutionnelle dans les prétoires apparaît plutôt comme une bonne nouvelle pour les spécialistes du contentieux constitutionnel : le procès constitutionnel s'ouvre aux justiciables; les avocats devront se former à la jurisprudence constitutionnelle; les publicistes y voient de nouvelles perspectives professionnelles... La controverse autour de la prétendue usurpation du juge des pouvoirs des autres institutions n'est sans doute pas achevée.

\section{Conclusion}

Le Professeur Chapus écrivait que : « ..., d'une façon générale, s'il est bien que les juges fassent de la morale, c'est à condition qu'ils en fassent le moins possible ». ${ }^{205} \mathrm{C}$ 'est en ce sens que Bergel affirmait qu'il "paraît préférable, avec Ripert, d'admettre qu'il faut fonder l'autorité du droit sur une conception positive, mais qu'il est nécessaire de faire appel à la morale dans l'élaboration de l'ordre juridique, même si on ne peut se contenter d'une idée générale et vague de justice ». ${ }^{206}$ On peut comprendre par là qu'il doit effectivement en faire, mais uniquement lorsque cela s'avère nécessaire. A l'instar du mythe de la séparation des pouvoirs, la séparation du droit et de la morale est introuvable. Le juge demeure un « usager » de la morale et est lui-même apprécié en référence à la moralité. De nombreux sujets nous ramènent à relation entre la morale et le droit. De plus en plus, les derniers refuges du « non-droit » cèdent les uns après les autres devant l'avalanche de normes et l'envie toujours plus pressante de combler ces « vides juridiques ». En prenant pied dans tous les domaines de la vie sociale, économique et domestique, la loi a étendu d'autant la place et le rôle du juriste dans la société. Le juriste est désormais sommé de donner son avis

203 TROPER (M.), « La liberté de l'interprète », https://www.senat.fr/colloques/office_du_juge/office_du_juge3.html consulté le 4 juillet 2017.

204 Le juge constitutionnel procède, dans l'intérêt de l'État de droit, à un élargissement de son domaine de contrôle Confère, HOUNAKE (K.), Les juridictions constitutionnelles dans les démocraties émergentes de l'Afrique noire francophone : Les cas du Bénin, du Gabon, du Niger, du Sénégal et du Togo, Thèse, Université de Lomé, 2012, p. 98.

205 Droit administratif général, tome I, 15e éd., no 910, p. 710.

206 BERGEL (J.L.), Théorie générale du droit, Dalloz, 2012, p. 52. 
sur...le mariage homosexuel, l'interdiction du clonage, l'expérimentation sur l'embryon, l'admission de l'euthanasie, la nécessité ou non de faire application du principe de précaution, etc. $^{207}$

Le juge est souvent confronté à des situations délicates lorsqu'il doit traiter des litiges dont le trame de fond renvoie à des valeurs de société. Comme le démontre Mbongo dans La qualité des décisions de justice, le juge est souvent saisi des affaires portant sur « les mauvaises vies ». Aussi, note-t-il que dans les affaires relatives aux ingérences commises dans le droit au respect de la vie privée par des législations non-permissives ou pénalisantes de certaines orientations sexuelles, ${ }^{208}$ autrement dit le contentieux des mauvaises vies, la sensibilité du juge semble avoir été initialement définie à partir de la jurisprudence Dudgeon c. Royaume-Uni rendue en 1981, à travers notamment cette idée que la seule circonstance que des membres de la Société puissent être choqués, offensés ou perturbés d'apprendre que d'autres avaient une orientation homosexuelle, une inclination sadomasochiste, alors que selon lui, cet argument ne suffit pas à justifier de sanctions ou de menaces de sanctions d'adultes consentants. ${ }^{209}$

Comment rester dans son rôle sans usurper les prérogatives des autres institutions? Il serait judicieux que le juge dans sa décision, dise d'abord le droit et qu'il sélectionne la norme juridique devant être appliquée au corpus de faits qui lui sont soumis par les parties. Le choix de cette norme doit donc nécessairement s'opérer en cohérence avec les demandes de justice qui lui sont présentées et, surtout, avec les faits de l'espèce. Cela suppose une interprétation des éléments constitutifs de la norme, surtout si le juge se trouve amené à utiliser ce qui est convenu d'appeler une notion juridique à contenu variable, dont la détermination est confiée au juge lui-même. Tout compte fait, dans une perspective de sécurité juridique, seule va compter la cohérence sur laquelle le juge se base pour rendre sa décision. N'est-ce pas là ce que veut signifier Atias par ce propos : " au commencement de l'acte juridictionnel, il y a inévitablement une intuition. Elle dépend assurément des convictions personnelles du juge [...] cette intuition première peut devenir préjugé, elle ne peut être qu'un guide [...] le choix doit être alors celui d'une justification aussi explicite que possible »??

207 A travers la bioéthique, l'évolution des rapports du droit et de la morale conduit à transformer le juriste face à la norme. Parce que le droit de la bioéthique touche à l'intime, aux croyances des individus sur les thèmes essentiels pour l'humain, toute personne doit pouvoir donner son avis sur les options à retenir, sachant que, au nom de la démocratie, les choix sociaux relèvent du seul législateur. Voir, FEUILLET (B.), « La place du juriste dans l'élaboration des lois bioéthiques », in Actes du Colloque « La place du juriste face à la norme », Dalloz, 2012, p. 67.

208 Homosexualité, sadomasochisme...

209 « La Cour européenne des droits de l'homme a-t-elle une philosophie morale? » in La qualité des décisions de justice, op. cit.

210 Cité par TARLET (F.), dans « Roger Latournerie, 'juge du juste’ », RFDA 2017, p. 383. 